\title{
Multipliers on Generalized Mixed Norm Sequence Spaces
}

\author{
Oscar Blasco and Carme Zaragoza-Berzosa \\ Departamento de Análisis Matemático, Universidad de Valencia, Burjassot, 46100 Valencia, Spain
}

Correspondence should be addressed to Carme Zaragoza-Berzosa; carme.zaragoza@uv.es

Received 7 November 2013; Revised 13 January 2014; Accepted 27 January 2014; Published 3 April 2014

Academic Editor: Fasma Diele

Copyright (c) 2014 O. Blasco and C. Zaragoza-Berzosa. This is an open access article distributed under the Creative Commons Attribution License, which permits unrestricted use, distribution, and reproduction in any medium, provided the original work is properly cited.

Given $1 \leq p, q \leq \infty$ and sequences of integers $\left(n_{k}\right)_{k}$ and $\left(n_{k}^{\prime}\right)_{k}$ such that $n_{k} \leq n_{k}^{\prime} \leq n_{k+1}$, the generalized mixed norm space $\ell^{\mathcal{J}}(p, q)$ is defined as those sequences $\left(a_{j}\right)_{j}$ such that $\left(\left(\sum_{j \in I_{k}}\left|a_{j}\right|^{p}\right)^{1 / p}\right)_{k} \in \ell^{q}$ where $I_{k}=\left\{j \in \mathbb{N}_{0}\right.$ s.t. $\left.n_{k} \leq j<n_{k}^{\prime}\right\}, k \in \mathbb{N}_{0}$. The necessary and sufficient conditions for a sequence $\lambda=\left(\lambda_{j}\right)_{j}$ to belong to the space of multipliers $\left(\ell^{\mathcal{J}}(r, s), \ell^{\mathcal{F}}(u, v)\right)$, for different sequences $\mathscr{I}$ and $\mathscr{J}$ of intervals in $\mathbb{N}_{0}$, are determined.

\section{Introduction}

Let $\mathcal{S}$ be the space of complex valued sequences with the locally convex vector topology given by means of the seminorms $p_{j}(\lambda)=\left|\lambda_{j}\right|$ where $\lambda=\left(\lambda_{j}\right)_{j \in \mathbb{N}_{0}}$. Given two Banach spaces $A, B$ continuously contained in $\mathcal{S}$, we write $(A, B)$ for the space of multipliers from $A$ into $B$. More precisely,

$$
(A, B)=\left\{\lambda=\left(\lambda_{j}\right)_{j}:\left(\lambda_{j} a_{j}\right)_{j} \in B ; \forall\left(a_{j}\right)_{j} \in A\right\} .
$$

We will use the notation $\operatorname{supp}(a)=\left\{n \in \mathbb{N}_{0}: a_{n} \neq 0\right\}$ and $\lambda * a$ for the sequence $\left(\lambda_{j} a_{j}\right)_{j \in \mathbb{N}_{0}}$ where $\lambda=\left(\lambda_{j}\right)_{j \in \mathbb{N}_{0}}$ and $a=\left(a_{j}\right)_{j \in \mathbb{N}_{0}}$.

Of course for the classical $\ell^{p}$ spaces, one easily sees that $\left(\ell^{p_{1}}, \ell^{p_{2}}\right)=\ell^{p}$ where $1 / p=\left(1 / p_{2}-1 / p_{1}\right)^{+}$. We use the notation $p_{2} \ominus p_{1}=p$ to mean $1 / p_{2} \ominus p_{1}=1 / p_{2}-1 / p_{1}$ whenever $p_{1}>p_{2}$ and $p=\infty$ whenever $p_{1} \leq p_{2}$.

The above result can be extended (see [1]) to the class of mixed norm sequence spaces, denoted $\ell(p, q)$, which are defined by the condition

$$
\left(\sum_{n=0}^{\infty}\left(\sum_{2^{n}-1 \leq k<2^{n+1}-1}\left|a_{k}\right|^{p}\right)^{q / p}\right)^{1 / q}<\infty .
$$

Theorem 1. Let $1 \leq r, s, u, v \leq \infty$. Then

$$
(\ell(r, s), \ell(u, v))=\ell(u \ominus r, v \ominus s) .
$$

In particular, the Köthe dual of $\ell(p, q)$, defined by $\left(\ell(p, q), \ell^{1}\right)$, becomes $\ell\left(p^{\prime}, q^{\prime}\right)$ for $1 \leq p, q<\infty$ and $1 / p+$ $1 / p^{\prime}=1 / q+1 / q^{\prime}=1$.

Also multipliers between sequence spaces given by Taylor coefficients of holomorphic functions in the disk have been deeply studied in the literature. Since the time of Hardy and Littlewood, mixed norm and related spaces have been used to study function spaces on the unit disk and later to study multipliers between such spaces. Special emphasis has been put on the case where the spaces $A$ and $B$ correspond to the sequence space of Taylor coefficient of analytic functions such as Hardy spaces, Bergman spaces, mixed norm spaces of analytic functions, and so forth. The theory of Hardy spaces and mixed norm spaces of analytic functions was originated in the work of Hardy and Littlewood (see $[2,3]$ ) who implicitly considered the spaces $H(p, q, \alpha)$ of functions $f \in \mathscr{H}(\mathbb{D})$ such that

$$
\left(\int_{0}^{1}(1-r)^{q \alpha-1} M_{p}^{q}(f, r) d r\right)^{1 / q}<\infty .
$$

Their work on these spaces was continued by Flett and Sledd (see $[4-8]$ ) and later on by Pavlović (see $[9,10])$. Multipliers on Hardy spaces were in fashion for a long time and much work was done on them and related spaces. However nowadays complete descriptions of multipliers between Hardy spaces $\left(H^{p}, H^{q}\right)$ for certain values of $p$ and $q$ remain still 
open. The reader is referred to the surveys (see $[11,12]$ ) for lots of results and references. Also many results on multipliers between mixed norm spaces of analytic functions have been established in the last decades (see [13-15] and references thereby). For such a purpose, the use of solid spaces (sequence spaces whose norm depends only on the size of the coefficients), and in particular $\ell(p, q)$ spaces, is a rather important tool. It is worth mentioning that the smallest solid space contained or which contains one of classical Hardy, Bergman, and $H(p, q, \alpha)$ is actually $H(2, q, \alpha)$ for some values $p, q$, and $\alpha$ (see $[14,15])$ and this last space can be identified with certain weighted $\ell(2, q)$, due to Plancherel's theorem.

Another appearance of mixed norm spaces comes with the use of lacunary sequences, that is, $a=\left(a_{n}\right)$, such that $\operatorname{supp}(a) \subset\left\{n_{k}: k \in \mathbb{N}_{0}\right\}$ for a sequence of integers satisfying $\inf n_{k+1} / n_{k}=\lambda>1$. Recently (see [16]) the description of the Taylor coefficient of analytic functions $F(z)=\sum_{k=0}^{\infty} b_{k} z^{n_{k}}$, where $n_{k}$ is a lacunary sequence, belonging to the weighted Bergman-Besov space $B^{1}(\rho)$ has been achieved under certain conditions on the weight. It corresponds again with certain weighted $\ell(2,1)$.

In this paper, we consider families of intervals $\mathscr{I}=\left\{I_{k}\right.$ : $\left.k \in \mathbb{N}_{0}\right\}$ where $I_{k}=\left\{j \in \mathbb{N}_{0}\right.$ s.t. $\left.n_{k} \leq j<n_{k}^{\prime}\right\}$ for some increasing sequences $\left(n_{k}\right)_{k}$ and $\left(n_{k}^{\prime}\right)$ such that $n_{k}<n_{k}^{\prime} \leq n_{k+1}$ and we use the notation $\Lambda_{\mathscr{I}}=\cup I_{k}$. We will introduce the spaces $\ell^{\mathcal{I}}(p, q)$ given by sequences $a=\left(a_{j}\right)_{j \in \Lambda_{\mathscr{I}}}$ verifying

$$
\left(\left(\sum_{j \in I_{k}}\left|a_{j}\right|^{p}\right)^{1 / p}\right)_{k} \in \ell^{q}
$$

and the obvious modifications for $p=\infty$ or $q=\infty$.

In particular, $\ell(p, q)=\ell^{\mathcal{I}}(p, q)$ for $I_{k}=\left[2^{k}-1,2^{k+1}-\right.$ 1) $\cap \mathbb{N}_{0}$. Also a lacunary sequence $a=\left(a_{n}\right)_{n}$ corresponds to $\operatorname{supp}(a) \subseteq \Lambda_{\mathscr{I}}$ where $\mathscr{I}=\left\{I_{k}: k \in \mathbb{N}_{0}\right\}$ with $I_{k}=\left\{n_{k}\right\}$ (that is, $\left.n_{k}^{\prime}=n_{k}+1\right)$ for some $\inf _{k} n_{k+1} / n_{k}=\lambda>1$.

We will give the necessary and sufficient conditions for a sequence $\lambda=\left(\lambda_{j}\right)_{j}$ to belong to the multiplier space $\left(\ell^{\mathscr{I}}(r, s), \ell^{\mathscr{f}}(u, v)\right)$ whenever $\Lambda_{\mathscr{I}}=\Lambda_{\mathscr{f}}$. We also get some applications to multipliers between certain weighted mixed norm spaces of analytic functions. The paper is organized as follows. Section 2 contains the definitions and first properties of the spaces $\ell^{\mathscr{I}}(p, q)$, studying inclusions between them and conditions for coincidence results $\ell^{\mathscr{I}}(p, q)=\ell^{\mathscr{F}}(p, q)$. Section 3 contains the main result, which is split into three subsections: the case where intervals in $\mathscr{J}$ are union of intervals in $\mathscr{I}$, to be denoted $\mathscr{I} \leq \mathscr{J}$, the case where for each $I \in \mathscr{I}$ there exists $J \in \mathscr{J}$ such that either $I \subseteq J$ or $J \subseteq I$, and finally the case where there exists $(I, J) \in \mathscr{I} \times \mathscr{J}$ such that $I \cap J \neq \emptyset$ and $I \cap J \notin \mathscr{I} \cup \mathscr{J}$. In Section 4, we include some application to multipliers on spaces of analytic functions and extend some recent result on weighted Bergman-Besov classes.

From now on, we will write $A \approx B$ whenever there exists $C>0$ such that $C^{-1} A \leq B \leq C A$ and, as usual, \#I stands for the cardinal of $I, 1 / p+1 / p^{\prime}=1$ for $1 \leq p \leq \infty$ and also $C$ denotes a constant that may vary from line to line.

\section{Generalized Mixed Norm Spaces}

Definition 2. Let $1 \leq p, q \leq \infty$ and let $\mathscr{I}$ be a collection of disjoint intervals in $\mathbb{N}_{0}$, say $I_{k}=\mathbb{N}_{0} \cap\left[n_{k}, n_{k}^{\prime}\right)$, where $n_{k}<$ $n_{k}^{\prime} \leq n_{k+1}$. One sets $\Lambda_{\mathscr{I}}=\cup_{k \in \mathbb{N}_{0}} I_{k}$. One writes $\ell^{\mathcal{I}}(p, q)$ for the space of sequences $\left(a_{n}\right)_{n \in \Lambda_{\mathcal{F}}}$ verifying

$$
\left(\left(\sum_{j \in I_{k}}\left|a_{j}\right|^{p}\right)^{1 / p}\right)_{k} \in \ell^{q} .
$$

This space becomes a Banach space under the norm

$$
\|a\|_{p, q}^{\mathcal{I}}=\left(\sum_{k=0}^{\infty}\left(\sum_{j \in I_{k}}\left|a_{j}\right|^{p}\right)^{q / p}\right)^{1 / q}
$$

with the obvious modifications for $p=\infty$ or $q=\infty$.

Remark 3. Of course $\ell^{\mathcal{I}}(p, p)=\left\{\left(a_{n}\right)_{n \in \Lambda_{\mathcal{I}}}:\left(\sum_{n}\left|a_{n}\right|^{p}\right)^{1 / p}<\right.$ $\infty\}$. In particular, $\ell^{\mathscr{I}}(p, p)=\ell^{p}$ whenever $\Lambda_{\mathscr{I}}=\mathbb{N}_{0}$.

An elementary approach, using Hölder's inequality, leads to the duality

$$
\ell^{\mathcal{I}}(p, q)^{*}=\ell^{\mathscr{I}}\left(p^{\prime}, q^{\prime}\right)
$$

for $1 \leq p, q<\infty$ and $1 / p+1 / p^{\prime}=1 / q+1 / q^{\prime}=1$.

Remark 4. It is clear that $\left(a_{j}\right)_{j} \in \ell^{\mathcal{I}}(p, q) \Leftrightarrow\left(a_{j}^{p}\right)_{j} \in \ell^{\mathscr{I}}(1$, $q / p)$ in the case $p<q$ and also $\left(a_{j}\right)_{j} \in \ell^{\mathcal{I}}(p, q) \Leftrightarrow\left(a_{j}^{q}\right)_{j} \in$ $\ell^{\mathscr{I}}((p / q), 1)$ in the case $p>q$.

Moreover, for $a^{p}=\left(a_{j}^{p}\right)_{j}$,

$$
\|a\|_{p, q}^{\mathcal{I}}=\left(\left\|a^{p}\right\|_{1, q / p}^{\mathcal{I}}\right)^{1 / p}=\left(\left\|a^{q}\right\|_{p / q, 1}^{\mathcal{I}}\right)^{1 / q} .
$$

Remark 5. Let $a \in \ell^{\mathcal{I}}(p, q)$.

(i) If $\mathscr{I}^{\prime}$ is a subcollection of intervals in $\mathscr{I}$, then $\|a\|_{p, q}^{\mathscr{I}^{\prime}} \leq$ $\|a\|_{p, q}^{\mathscr{I}}$.

(ii) If $\mathscr{I}=\mathscr{I}^{\prime} \cup \mathscr{I}^{\prime \prime}$ for two disjoint collections $\mathscr{I}^{\prime}$ and $\mathscr{I}^{\prime \prime}$, then $\|a\|_{p, q}^{\mathcal{I}}=\left(\left(\|a\|_{p, q}^{\mathcal{F}^{\prime}}\right)^{q}+\left(\|a\|_{p, q}^{\mathcal{J}^{\prime \prime}}\right)^{q}\right)^{1 / q}$.

We would like to analyze the embedding between $\ell^{\mathcal{I}}\left(p_{1}, q_{1}\right)$ and $\ell^{\mathscr{I}}\left(p_{2}, q_{2}\right)$.

Proposition 6. Let $\mathscr{I}$ be a collection of disjoint intervals in $\mathbb{N}_{0}$ and let $1 \leq p_{1}, p_{2}, q \leq \infty$ with $p_{1} \neq p_{2}$. Then $\ell^{\mathscr{I}}\left(p_{1}, q\right)=$ $\ell^{\mathcal{I}}\left(p_{2}, q\right)$ (with equivalent norms) if and only if

$$
\sup _{k \in \mathbb{N}_{0}} \# I_{k}<\infty \text {. }
$$

In particular, if $\sup _{k \in \mathbb{N}_{0}} \# I_{k}<\infty$, then

$$
\ell^{\mathscr{I}}(p, q)=\left\{\left(a_{n}\right)_{n \in \Lambda_{\mathcal{F}}}:\left(\sum_{n}\left|a_{n}\right|^{q}\right)^{1 / q}<\infty\right\} .
$$


Proof. $\Rightarrow$ ) Assume, for instance, $p_{1}<p_{2}$ and that $\|a\|_{p_{1}, q}^{\mathcal{F}} \approx$ $\|a\|_{p_{2}, q}^{\mathscr{J}}$ for all $a$ supported in $\Lambda_{\mathscr{F}}$. Hence, taking $a=\chi_{I_{k}}$, one concludes that $\left(n_{k}^{\prime}-n_{k}\right)^{1 / p_{1}-1 / p_{2}} \leq C$ for any $k$. Hence $\sup _{k} \# I_{k}<\infty$.

$\Leftarrow)$ Note that $\# I_{k}=\left(n_{k}^{\prime}-n_{k}\right)$ and assume $M=\sup _{k}\left(n_{k}-\right.$ $\left.n_{k}^{\prime}\right)$. Then

$$
\begin{aligned}
\|a\|_{p_{1}, q}^{\mathcal{G}} & =\left(\sum_{k=0}^{\infty}\left(\sum_{j \in I_{k}}\left|a_{j}\right|^{p_{1}}\right)^{q / p_{1}}\right)^{1 / q} \\
& \approx\left(\sum_{k=0}^{\infty}\left(\sum_{j \in I_{k}}\left|a_{j}\right|^{p_{2}}\right)^{q / p_{2}}\right)^{1 / q}=\|a\|_{p_{2}, q}^{\mathcal{G}}
\end{aligned}
$$

since $\|\cdot\|_{p_{1}} \approx\|\cdot\|_{p_{2}}$ in $\mathbb{C}^{M}$.

Proposition 7. Let $1 \leq p_{1}, q_{1}, p_{2}, q_{2} \leq \infty$ and let $\mathscr{I}$ be $a$ collection of disjoint intervals in $\mathbb{N}_{0}$ with $\sup _{k} \# I_{k}=\infty$.

Then $\ell^{\mathcal{I}}\left(p_{1}, q_{1}\right) \subseteq \ell^{\mathcal{J}}\left(p_{2}, q_{2}\right)$ if and only if $p_{1} \leq p_{2}$ and $q_{1} \leq q_{2}$.

Proof. $\Rightarrow$ ) Assume that there exists $C>0$ such that $\|a\|_{p_{2}, q_{2}}^{\mathcal{g}} \leq$ $C\|a\|_{p_{1}, q_{1}}^{\mathscr{g}}$ for all $a$ supported in $\Lambda_{\mathcal{F}}$. Hence, taking $k \in \mathbb{N}_{0}$ and $a=\chi_{I_{k}}$, one concludes that $\left(\# I_{k}\right)^{1 / p_{2}-1 / p_{1}} \leq C$. Hence $p_{1} \leq p_{2}$. Let $N \in \mathbb{N}_{0}$ and consider $a=\sum_{k=1}^{N} \chi_{n_{k}}$. Applying the above inequality, we obtain $N^{1 / q_{2}-1 / q_{1}} \leq C$. Therefore, $q_{1} \leq q_{2}$.

$\Leftarrow)$ Let us denote

$$
\ell^{q}\left(\ell^{p}\right)=\left\{\left(x_{k}\right)_{k \in \mathbb{N}_{0}}: x_{k} \in \ell^{p},\left(\sum_{k=0}^{\infty}\left\|x_{k}\right\|_{\ell^{p}}^{q}\right)^{1 / q}<\infty\right\} .
$$

Hence the mapping

$$
\left(a_{n}\right)_{n \in \mathbb{N}_{0}} \longrightarrow\left(\left(a_{j}\right)_{j \in I_{k}}\right)_{k \in \mathbb{N}_{0}}
$$

is an isometric embedding from $\ell^{\mathcal{J}}(p, q)$ into $\ell^{q}\left(\ell^{p}\right)$. Taking into account that $\ell^{r_{1}}(E) \subseteq \ell^{r_{2}}(E)$ for any Banach space $E$ and $r_{1} \leq r_{2}$, we conclude that

$$
\ell^{\mathcal{F}}\left(p, q_{1}\right) \subseteq \ell^{\mathcal{F}}\left(p, q_{2}\right), \quad \ell^{\mathcal{F}}\left(p_{1}, q\right) \subseteq \ell^{\mathcal{F}}\left(p_{2}, q\right) .
$$

Therefore

$$
\ell^{\mathcal{I}}\left(p_{1}, q_{1}\right) \subseteq \ell^{\mathcal{I}}\left(p_{2}, q_{1}\right) \subseteq \ell^{\mathcal{I}}\left(p_{2}, q_{2}\right) .
$$

We would like to analyze the embedding between $\ell^{\mathcal{I}}(p$, $q)$ and $\ell^{\mathscr{f}}(p, q)$ for $\mathscr{I} \neq \mathscr{f}$ whenever $\Lambda_{\mathscr{I}}=\Lambda_{\mathscr{f}}$.

Proposition 8. Let $\mathscr{I}=\left\{I_{l}: l \in \mathbb{N}_{0}\right\}$ and $\mathscr{J}=\left\{J_{k}: k \in \mathbb{N}_{0}\right\}$. If $\Lambda_{\mathscr{I}}=\Lambda_{\mathscr{f}}, p \leq q$ (respect. $\left.q \leq p\right)$, and $\sup _{k} \# I_{k}<\infty$ (respect. $\left.\sup _{l} \# I_{l}<\infty\right)$, then

$$
\ell^{\mathscr{I}}(p, q) \subseteq \ell^{\mathscr{F}}(p, q)\left(\text { respect. } \ell^{\mathscr{F}}(p, q) \subseteq \ell^{\mathscr{I}}(p, q)\right) .
$$

Proof. Proposition 6 gives $\ell^{\mathscr{F}}(p, q)=\ell^{\mathscr{f}}(q, q)$, and clearly $\ell^{\mathscr{F}}(q, q)=\ell^{\mathcal{F}}(q, q)$. Then the result follows using $\ell^{\mathcal{F}}(p, q) \subseteq$ $\ell^{\mathcal{J}}(q, q)$ whenever $p \leq q$.

Let us mention another particular case where they coincide.

Proposition 9. Let $\mathscr{I}$ be such that $I_{k}=\left[n_{k}, n_{k}^{\prime}\right) \cap \mathbb{N}_{0}$ with $n_{2 k}^{\prime}=n_{2 k+1}$ and define

$$
\mathscr{J}=\left\{J_{k}=I_{2 k} \cup I_{2 k+1}: k \in \mathbb{N}_{0}\right\} .
$$

Then $\ell^{\mathcal{I}}(p, q)=\ell^{\mathcal{F}}(p, q)$.

Proof. Note that $J_{k}=I_{2 k} \cup I_{2 k+1}$ is again an interval in $\mathbb{N}_{0}$. Using that $(a+b)^{\alpha} \leq C_{\alpha}\left(a^{\alpha}+b^{\alpha}\right)$ for $a, b, \alpha>0$, then

$$
\begin{aligned}
\|a\|_{p, q}^{\mathcal{g}} & =\left(\sum_{k=0}^{\infty}\left(\sum_{j \in \epsilon_{k}}\left|a_{j}\right|^{p}\right)^{q / p}\right)^{1 / q} \\
& =\left(\sum_{k=0}^{\infty}\left(\sum_{j \in I_{2 k}}\left|a_{j}\right|^{p}+\sum_{j \in I_{2 k+1}}\left|a_{j}\right|^{p}\right)^{q / p}\right)^{1 / q} \\
& \leq C\left(\sum_{k=0}^{\infty}\left(\sum_{j \in I_{2 k}}\left|a_{j}\right|^{p}\right)^{q / p}+\sum_{k=0}^{\infty}\left(\sum_{j \in I_{2 k+1}}\left|a_{j}\right|^{p}\right)^{q / p}\right)^{1 / q} \\
& \leq C\|a\|_{p, q}^{\mathcal{F}} .
\end{aligned}
$$

On the other hand, using now $\left(a^{\beta}+b^{\beta}\right) \leq C_{\beta}(a+b)^{\beta}$ for $a, b, \beta>0$,

$$
\begin{aligned}
\|a\|_{p, q}^{\mathcal{F}} & =\left(\sum_{k=0}^{\infty}\left(\sum_{j \in I_{2 k}}\left|a_{j}\right|^{p}\right)^{q / p}+\left(\sum_{j \in I_{2 k+1}}\left|a_{j}\right|^{p}\right)^{q / p}\right)^{1 / q} \\
& \leq C^{\prime}\left(\sum_{k=0}^{\infty}\left(\sum_{j \in I_{2 k} \cup I_{2 k+1}}\left|a_{j}\right|^{p}\right)^{q / p}\right)^{1 / q} \\
& \leq C^{\prime}\|a\|_{p, q}^{\mathcal{g}} .
\end{aligned}
$$

The previous idea is easily generalized using the following definition.

Definition 10. Let $\mathscr{I}:=\left\{I_{l}: l \in \mathbb{N}_{0}\right\}$ and $\mathscr{J}:=\left\{J_{k}: k \in \mathbb{N}_{0}\right\}$. One says that $\mathscr{I} \leq \mathscr{J}$ if the following conditions hold:

(i) $\Lambda_{\mathscr{I}}=\Lambda_{\mathscr{F}}$;

(ii) $F_{k}=F_{k}(\mathscr{I}, \mathscr{f}):=\left\{l \in \mathbb{N}_{0}: I_{l} \subseteq J_{k}\right\} \neq \emptyset$ for all $k \in \mathbb{N}_{0}$;

(iii) $J_{k}=\cup_{l \in F_{k}} I_{l}$ for all $k \in \mathbb{N}_{0}$. 
Proposition 11. Let $1 \leq p, q \leq \infty$ and $\mathscr{I} \leq \mathscr{J}$. Then

(i) $\ell^{\mathscr{I}}(p, q) \subseteq \ell^{\mathcal{F}}(p, q)$ for $p \leq q$;

(ii) $\ell^{\mathcal{F}}(p, q) \subseteq \ell^{\mathcal{F}}(p, q)$ for $q \leq p$.

Moreover, the embeddings above are of norm 1.

Proof. (i) Case $q=\infty$ : let $a \in \ell^{\mathcal{f}}(p, \infty)$ and $l \in \mathbb{N}_{0}$. We know that there is $k$ such that $I_{l} \subseteq J_{k}$. Hence

$$
\left(\sum_{n \in I_{l}}\left|a_{n}\right|^{p}\right)^{1 / p} \leq\left(\sum_{n \in J_{k}}\left|a_{n}\right|^{p}\right)^{1 / p} \leq\|a\|_{p, \infty}^{\mathcal{F}} .
$$

This gives $\|a\|_{p, \infty}^{\mathscr{I}} \leq\|a\|_{p, \infty}^{\mathscr{F}}$.

The case $p=1$ : let $a \in \ell^{\mathscr{f}}(1, q)$ and $q \geq 1$. Therefore

$$
\begin{aligned}
\left(\|a\|_{1, q}^{\mathcal{g}}\right)^{q} & =\sum_{k}\left(\sum_{l \in F_{k}} \sum_{n \in I_{l}}\left|a_{n}\right|\right)^{q} \\
& \geq \sum_{k} \sum_{l \in F_{k}}\left(\sum_{n \in I_{l}}\left|a_{n}\right|\right)^{q}=\left(\|a\|_{1, q}^{\mathcal{F}}\right)^{q} .
\end{aligned}
$$

The case $1<p \leq q<\infty$ follows using (9) and the previous one.

(ii) The case $p=\infty$ : let $a \in \ell^{\mathscr{I}}(\infty, q)$. Then

$$
\begin{aligned}
\|a\|_{\infty, q}^{\mathcal{g}} & =\left(\sum_{k} \sup _{l \in F_{k}}\left(\sup _{n \in I_{l}}\left|a_{n}\right|\right)^{q}\right)^{1 / q} \\
& \leq\left(\sum_{k} \sum_{l \in F_{k}}\left(\sup _{n \in I_{l}}\left|a_{n}\right|\right)^{q}\right)^{1 / q}=\|a\|_{\infty, q}^{\mathcal{I}} .
\end{aligned}
$$

To cover the remaining cases, from (9), we simply need to show that $\ell^{\mathcal{I}}(p, 1) \subseteq \ell^{\mathscr{I}}(p, 1)$ for $p \geq 1$. Now observe that

$$
\begin{aligned}
\|a\|_{p, 1}^{\mathscr{g}} & =\sum_{k}\left(\sum_{l \in F_{k}} \sum_{n \in I_{l}}\left|a_{n}\right|^{p}\right)^{1 / p} \\
& =\sum_{k}\left(\sum_{l \in F_{k}}\left\|a \chi_{I_{l}}\right\|_{p}^{p}\right)^{1 / p} \\
& \leq \sum_{k} \sum_{l \in F_{k}}\left\|a \chi_{I_{l}}\right\|_{p}=\sum_{l}\left(\sum_{n \in I_{l}}\left|a_{n}\right|^{p}\right)^{1 / p} \\
& =\|a\|_{p, 1}^{\mathcal{I}} .
\end{aligned}
$$

Theorem 12. Let $\mathscr{I} \leq \mathscr{J}$ and $1 \leq p, q \leq \infty$ with $p \neq q$.

$\ell^{\mathscr{I}}(p, q)=\ell^{\mathscr{F}}(p, q)$ (with equivalent norms) if and only if $\sup _{k} \# F_{k}<\infty$.

Proof. $\Rightarrow$ ) Assume that $\|a\|_{p, q}^{\mathscr{g}} \approx\|a\|_{p, q}^{\mathscr{I}}$ for all $a$ finitely supported Let $k \in \mathbb{N}_{0}$ and define

$$
a^{(k)}=\sum_{l \in F_{k}}\left(\# I_{l}\right)^{-1 / p} \chi_{I_{l}} .
$$

Then $\|a\|_{p, q}^{\mathscr{g}}=\left(\# F_{k}\right)^{1 / p}$ and $\|a\|_{p, q}^{\mathscr{I}}=\left(\# F_{k}\right)^{1 / q}$.
One concludes that $C_{2} \leq\left(\# F_{k}\right)^{1 / p-1 / q} \leq C_{1}$ which implies, in the case $p \neq q$, $\sup _{k \in \mathbb{N}_{0}}\left(\# F_{k}\right)<\infty$.

$\Leftarrow)$ Case $p<q$. From Proposition 11, we only need to show $\ell^{\mathcal{I}}(p, q) \subseteq \ell^{\mathscr{f}}(p, q)$. Using now Hölder's inequality for $q / p>$ 1 ,

$$
\begin{aligned}
\left(\sum_{n \in J_{k}}\left|a_{n}\right|^{p}\right)^{1 / p} & \leq\left(\sum_{l \in F_{k}} \sum_{n \in I_{l}}\left|a_{n}\right|^{p}\right)^{1 / p} \\
& \leq\left(\sum_{l \in F_{k}}\left(\sum_{n \in I_{l}}\left|a_{n}\right|^{p}\right)^{q / p}\right)^{1 / q}\left(\# F_{k}\right)^{1 / p \ominus q} .
\end{aligned}
$$

Therefore, if $M=\sup _{k} F_{k}$, we have

$$
\begin{aligned}
\|a\|_{p, q}^{\mathcal{J}} & =\left(\sum_{k=0}^{\infty}\left(\sum_{n \in J_{k}}\left|a_{n}\right|^{p}\right)^{q / p}\right)^{1 / q} \\
& \leq M^{1 / p \ominus q}\left(\sum_{k \in \mathbb{N}_{0}} \sum_{l \in F_{k}}\left(\sum_{n \in I_{l}}\left|a_{n}\right|^{p}\right)^{q / p}\right)^{1 / q} \\
& =M^{1 / p \ominus q}\left(\sum_{l \in \mathbb{N}_{0}}\left(\sum_{n \in I_{l}}\left|a_{n}\right|^{p}\right)^{q / p}\right)^{1 / q} \\
& =M^{1 / p \ominus q}\|a\|_{p, q}^{\mathcal{F}} .
\end{aligned}
$$

Case $p>q$. Using again Proposition 11, we will show $\ell^{\mathscr{f}}(p, q) \subseteq \ell^{\mathscr{I}}(p, q)$. Using $1 / q=1 / q \ominus p+1 / p$,

$$
\begin{aligned}
\|a\|_{p, q}^{\mathcal{J}} & =\left(\sum_{l}\left\|a \chi_{I_{l}}\right\|_{p}^{q}\right)^{1 / q} \\
& =\left(\sum_{k} \sum_{l \in F_{k}}\left\|a \chi_{I_{l}}\right\|_{p}^{q}\right)^{1 / q} \\
& \leq\left(\sum_{k}\left(\sum_{l \in F_{k}}\left\|a \chi_{I_{l}}\right\|_{p}^{p}\right)^{q / p}\left(\# F_{k}\right)^{q / q \ominus p}\right)^{1 / q} \\
& \leq M^{1 / q \ominus p}\left(\sum_{k}\left(\sum_{n \in J_{k}}\left|a_{n}\right|^{p}\right)^{q / p}\right)^{1 / q} \\
& \leq M^{1 / q \ominus p}\|a\|_{p, q}^{\mathscr{g}} .
\end{aligned}
$$

Let us now exhibit an example where neither $\ell(p, q)^{\mathscr{I}} \subseteq$ $\ell^{\mathscr{F}}(p, q)$ nor $\ell^{\mathscr{f}}(p, q) \subseteq \ell^{\mathscr{I}}(p, q)$. 
Example 13. Let $1 \leq p<q<\infty$ and take $\mathscr{I}, \mathscr{J}$ as shown in Figure 1 with

$$
\begin{aligned}
& \operatorname{card}\left(I_{0}\right)=n_{1} \\
& \operatorname{card}\left(I_{1}\right)=\cdots=\operatorname{card}\left(I_{n_{1}}\right)=1 \\
& \operatorname{card}\left(I_{n_{1}+1}\right)=n_{2} \\
& \operatorname{card}\left(I_{n_{1}+2}\right)=\cdots=\operatorname{card}\left(I_{n_{1}+n_{2}+1}\right)=1 \\
& \operatorname{card}\left(I_{n_{1}+n_{2}+2}\right)=n_{3}, \ldots \\
& \operatorname{card}\left(J_{0}\right)=\cdots=\operatorname{card}\left(J_{n_{1}}\right)=1 \\
& \operatorname{card}\left(J_{n_{1}}\right)=n_{1} \\
& \operatorname{card}\left(J_{n_{1}+1}\right)=\cdots=\operatorname{card}\left(J_{n_{1}+n_{2}}\right)=1 \\
& \operatorname{card}\left(J_{n_{1}+n_{2}+1}\right)=n_{2}, \ldots
\end{aligned}
$$

Let us see that neither $\ell^{\mathscr{F}}(p, q) \subset \ell^{\mathscr{I}}(p, q)$ nor $\ell^{\mathscr{I}}(p, q) \subset$ $\ell^{\mathscr{F}}(p, q)$.

Taking

$$
\begin{aligned}
& a=(\overbrace{\beta_{1}, \ldots, \beta_{1}}^{n_{1}}, \overbrace{0, \ldots, 0}^{n_{1}}, \overbrace{\beta_{2}, \ldots, \beta_{2}}^{n_{2}}, 0, \ldots), \\
& b=(\underbrace{0, \ldots, 0}_{n_{1}}, \underbrace{\beta_{1}, \ldots, \beta_{1}}_{n_{1}}, \underbrace{0, \ldots, 0}_{n_{2}}, \beta_{2}, \ldots)
\end{aligned}
$$

we have

$$
\begin{gathered}
\|a\|_{p, q}^{\mathcal{I}}=\|b\|_{p, q}^{\mathcal{g}}=\left(\sum_{j} \beta_{j}^{q} n_{j}^{q / p}\right)^{1 / q}, \\
\|a\|_{p, q}^{\mathcal{g}}=\|b\|_{p, q}^{\mathcal{I}}=\left(\sum_{j} \beta_{j}^{q} n_{j}\right)^{1 / q} .
\end{gathered}
$$
Now

Then it is enough to consider $q>p$ and $\beta_{j}=n_{j}^{-1 / p} j^{-1 / q}$.

$$
\left(\sum_{j} \beta_{j}^{q} n_{j}^{q / p}\right)^{1 / q}=\left(\sum_{j} j^{-1}\right)^{1 / q}=\infty
$$

and, since $n_{j} \geq j$,

$$
\begin{aligned}
\left(\sum_{j} \beta_{j}^{q} n_{j}\right)^{1 / q} & =\left(\sum_{j} j^{-1} n_{j}^{1-q / p}\right)^{1 / q} \\
& \leq\left(\sum_{j} j^{-q / p}\right)^{1 / q}<\infty .
\end{aligned}
$$

Hence we have $a \in \ell^{\mathscr{F}}(p, q) \backslash \ell^{\mathscr{I}}(p, q)$ and $b \in \ell^{\mathscr{I}}(p, q) \backslash$ $\ell^{\mathscr{f}}(p, q)$.

We would like to explain a procedure to analyze the general case $\Lambda_{\mathscr{I}}=\Lambda_{\mathscr{g}}$.
Definition 14. Let $\mathscr{I}$ and $\mathscr{J}$ be families of disjoint intervals in $\mathbb{N}_{0}$ with $\Lambda_{\mathscr{I}}=\Lambda_{\mathscr{g}}$. For each $k \in \mathbb{N}_{0}$, one uses the notation, as above, $F_{k}=\left\{l \in \mathbb{N}_{0}: I_{l} \subseteq J_{k}\right\}$ which now might be empty. One also defines

$$
\widetilde{F}_{k}=\left\{l \in \mathbb{N}_{0}: J_{k} \cap I_{l} \neq \emptyset\right\} .
$$

We write $\phi$ and $\Phi$ for the mappings given by

$$
\phi(k)=\min \widetilde{F}_{k}, \quad \Phi(k)=\max \widetilde{F}_{k} .
$$

Similarly, interchanging $\mathscr{I}$ and $\mathscr{J}$, we define $G_{l}, \widetilde{G}_{l}, \psi(l)$, and $\psi(l)$.

Definition 15. One defines the "left" and "right" part of the interval $J_{k}$ by

$$
\check{J}_{k}=J_{k} \cap I_{\phi(k)}, \quad \widehat{J}_{k}=J_{k} \cap I_{\Phi(k)}
$$

and, denoting $J_{k}^{\prime}=\cup_{l \in F_{k}} I_{l}$ and $\widetilde{J}_{k}=\cup_{l \in \widetilde{F}_{k}} I_{l}$, one has

$$
\begin{gathered}
J_{k}^{\prime} \subseteq J_{k} \subseteq \widetilde{J}_{k}, \\
J_{k}=J_{k}^{\prime} \cup \widehat{J}_{k} \cup \check{J}_{k},
\end{gathered}
$$

where $J_{k}^{\prime}=\emptyset$ whenever $F_{k}=\emptyset$.

Similarly, interchanging $\mathscr{I}$ and $\mathscr{J}$, we consider $\check{I}_{l}, \widehat{I}_{l}, I_{l}^{\prime}$, and $\tilde{I}_{l}$

With this notation out of the way we can classify intervals in $\mathscr{J}$ into four different types (according to $\mathscr{I}$ ). Note that for each interval $J \in \mathscr{J}$ there are four possibilities: $J$ coincides with $I$ for some $I \in \mathscr{I}, J$ can be written as a union of at least two intervals in $\mathscr{I}, J$ is strictly contained into some interval $I \in \mathscr{I}$, or there exists $I \in \mathscr{I}$ which overlaps with $J$ and its complement $J^{c}$.

Therefore we decompose $\mathbb{N}_{0}$ into four disjoint sets defined as follows.

Definition 16. Let $\mathscr{I}$ and $\mathscr{J}$ be families of disjoint intervals in $\mathbb{N}_{0}$ with $\Lambda_{\mathscr{I}}=\Lambda_{\mathscr{g}}$. One introduces

$$
\begin{array}{r}
N_{\text {equal }}^{\mathscr{g}}=\left\{k \in \mathbb{N}_{0}: \#\left(\widetilde{F}_{k} \backslash F_{k}\right)=0, \# \widetilde{F}_{k}=1\right\}, \\
N_{\text {big }}^{\mathcal{g}}=\left\{k \in \mathbb{N}_{0}: \#\left(\widetilde{F}_{k} \backslash F_{k}\right)=0, \# \widetilde{F}_{k} \geq 2\right\}, \\
N_{\text {small }}^{\mathcal{g}}=\left\{k \in \mathbb{N}_{0}: \#\left(\widetilde{F}_{k} \backslash F_{k}\right)>0, \# \widetilde{F}_{k}=1\right\}, \\
N_{\text {inter }}^{\mathscr{g}}=\left\{k \in \mathbb{N}_{0}: \#\left(\widetilde{F}_{k} \backslash F_{k}\right)>0, \# \widetilde{F}_{k} \geq 2\right\} .
\end{array}
$$

We define the sets $N_{\text {equal }}^{\mathscr{I}}, N_{\text {big }}^{\mathscr{I}}, N_{\text {small }}^{\mathcal{I}}$, and $N_{\text {inter }}^{\mathscr{I}}$ similarly.

Remark 17. Using (38), we can also give a description of the sets above in terms of $\phi$ and $\Phi$ :

$$
\begin{aligned}
N_{\text {equal }}^{\mathcal{g}} & =\left\{k: \phi(k)=\Phi(k), J_{k}=I_{\phi(k)}\right\} . \\
N_{\text {big }}^{\mathcal{g}} & =\left\{k: \phi(k)<\Phi(k), J_{k}=\widetilde{J}_{k}\right\} . \\
N_{\text {small }}^{\mathcal{g}} & =\left\{k: \phi(k)=\Phi(k), J_{k} \mp I_{\phi(k)}\right\} . \\
N_{\text {inter }}^{\mathcal{g}} & =\left\{k: \phi(k)<\Phi(k), J_{k} \mp \widetilde{J}_{k}\right\} .
\end{aligned}
$$




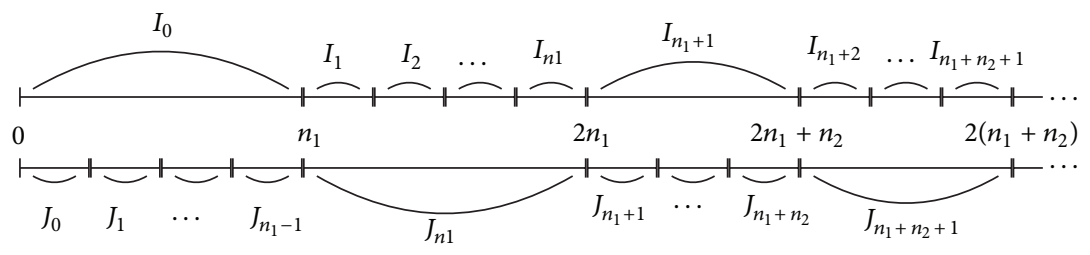

Figure 1

Using the above decomposition, we can generalize Propositions 8 and 11 and Theorem 12. Note that $\sup _{k} \# J_{k}<\infty$ implies $\sup _{k} \# \widetilde{F}_{k}<\infty$ and also that $\mathscr{I} \leq \mathscr{J}$ corresponds to the case where $N_{\text {inter }}^{\mathscr{q}} \cup N_{\text {small }}^{\mathscr{g}}=\emptyset$ or equivalently $\# \widetilde{G}_{l}=1$ for any $l \in \mathbb{N}_{0}$.

Theorem 18. Let $1 \leq p<q \leq \infty$ and $\mathscr{I}, \mathscr{J}$ be collections of intervals such that

$$
\ell^{\mathscr{I}}(p, q) \subseteq \ell^{\mathscr{F}}(p, q) \Longleftrightarrow \sup \left\{\# \widetilde{F}_{k} ; k \in \mathbb{N}_{0}\right\}<\infty .
$$

Proof. $\Rightarrow$ ) Arguing as in Theorem 12, for $k \in \mathbb{N}_{0}$ we consider

$$
a^{(k)}=\sum_{l \in \tilde{F}_{k}}\left(\#\left(I_{l} \cap J_{k}\right)\right)^{-1 / p} \chi_{I_{l} \cap J_{k}} .
$$

Hence

$$
\begin{aligned}
\left\|a^{(k)}\right\|_{p, q}^{\mathcal{g}} & =\left(\sum_{n \in J_{k}}\left|a_{n}\right|^{p}\right)^{1 / p} \\
& =\left(\sum_{l \in \widetilde{F}_{k}} \sum_{n \in I_{l} \cap J_{k}}\left|a_{n}\right|^{p}\right)^{1 / p}=\left(\# \widetilde{F}_{k}\right)^{1 / p}, \\
\left\|a^{(k)}\right\|_{p, q}^{\mathcal{F}} & =\left(\sum_{l \in \widetilde{F}_{k}}\left(\sum_{n \in I_{l} \cap J_{k}}\left|a_{n}\right|^{p}\right)^{q / p}\right)^{1 / q}=\left(\# \widetilde{F}_{k}\right)^{1 / q} .
\end{aligned}
$$

Therefore, using that $\left\|a^{(k)}\right\|_{p, q}^{\mathcal{g}} \leq C\left\|a^{(k)}\right\|_{p, q}^{\mathcal{I}}$ and $p<q$, we conclude that $\sup \left\{\# \widetilde{F}_{k} ; k \in \mathbb{N}_{0}\right\}<\infty$.

$\Leftarrow)$ Denote $\sup _{k}\left(\# F_{k}\right)=M \geq 0$ and let $k \in \mathbb{N}_{0}$.

Case $q=\infty$. If $k \in N_{\text {small }}^{\mathscr{g}} \cup N_{\text {equal }}^{\mathscr{g}}$, then

$$
\left(\sum_{n \in J_{k}}\left|a_{n}\right|^{p}\right)^{1 / p} \leq\left(\sum_{n \in I_{\phi(k)}}\left|a_{n}\right|^{p}\right)^{1 / p} \leq\|a\|_{p, \infty}^{\mathcal{F}} .
$$

If $k \in N_{\text {big }}^{\mathscr{J}} \cup N_{\text {inter }}^{\mathscr{J}}$, we have

$$
\begin{aligned}
\left(\sum_{n \in J_{k}}\left|a_{n}\right|^{p}\right)^{1 / p} & =\left(\sum_{l \in F_{k}} \sum_{n \in I_{l}}\left|a_{n}\right|^{p}+\sum_{n \in \breve{J}_{k} \cup \widehat{\cup}_{k}}\left|a_{n}\right|^{p}\right)^{1 / p} \\
& \leq\left(\sum_{l \in F_{k}} \sum_{n \in I_{l}}\left|a_{n}\right|^{p}\right)^{1 / p}+\left(\sum_{n \in I_{\phi(k)}}\left|a_{n}\right|^{p}\right)^{1 / p}
\end{aligned}
$$

$$
\begin{gathered}
+\left(\sum_{n \in I_{\Phi(k)}}\left|a_{n}\right|^{p}\right)^{1 / p} \\
\leq C\left(\sup _{l \in F_{k}}\left(\sum_{n \in I_{l}}\left|a_{n}\right|^{p}\right)^{1 / p}\right. \\
\left.\times\left(\# F_{k}\right)^{1 / p}+2\|a\|_{p, \infty}^{\mathcal{J}}\right) .
\end{gathered}
$$

This shows $\ell^{\mathcal{I}}(p, \infty) \subseteq \ell^{\mathscr{I}}(p, \infty)$.

Case $q<\infty$. Arguing as in Proposition 11, we simply show that $\ell^{\mathscr{I}}(1, q) \subseteq \ell^{\mathscr{F}}(1, q)$ for $q>1$.

Observe that

$$
\begin{aligned}
& \sum_{k \in N_{\text {small }}^{\mathcal{F}}}\left(\sum_{n \in J_{k}}\left|a_{n}\right|\right)^{q}
\end{aligned}
$$

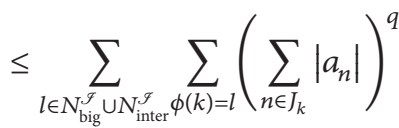

$$
\begin{aligned}
& \leq \sum_{l \in N_{\text {big }}^{\mathcal{Y} \cup N_{\text {inter }}^{\mathcal{S}}}}\left(\sum_{\phi(k)=l} \sum_{n \in J_{k}}\left|a_{n}\right|\right)^{q} \\
& =\sum_{l \in N_{\text {big }}^{\mathcal{F} \cup N_{\text {inter }}^{\mathcal{F}}}}\left(\sum_{n \in I_{l}}\left|a_{n}\right|\right)^{q} \leq\left(\|a\|_{1, q}^{\mathcal{G}}\right)^{q} .
\end{aligned}
$$

Also we have

$$
\begin{aligned}
& \sum_{k \in N_{\text {equal }}^{q} \cup N_{\text {big }}^{q}}\left(\sum_{n \in J_{k}}\left|a_{n}\right|\right)^{q} \\
& \leq \sum_{k \in N_{\text {equal }}^{q} \cup N_{\text {big }}^{q}}\left(\sum_{l \in F_{k}} \sum_{n \in I_{l}}\left|a_{n}\right|\right)^{q} \\
& \leq \sum_{k \in N_{\text {equal }}^{q} \text { l } N_{\text {big }}^{g}}\left(\# F_{k}\right)^{q-1} \sum_{l \in F_{k}}\left(\sum_{n \in I_{l}}\left|a_{n}\right|\right)^{q}
\end{aligned}
$$




$$
\begin{aligned}
& \leq M^{q-1} \sum_{k \in N_{\text {equal }}^{\mathcal{Y}} \cup N_{\text {big }}^{f} l \in F_{k}} \sum_{n \in I_{l}}\left(\sum_{n} \mid\right)^{q} \\
& \leq M^{q-1}\left(\|a\|_{1, q}^{\mathcal{F}}\right)^{q} .
\end{aligned}
$$

Finally

$$
\begin{aligned}
& \sum_{k \in N_{\text {inter }}^{q}}\left(\sum_{n \in J_{k}}\left|a_{n}\right|\right)^{q} \\
& \leq \sum_{k \in N_{\text {inter }}^{q}}\left(\sum_{l \in F_{k}} \sum_{n \in I_{l}}\left|a_{n}\right|+\sum_{n \in \breve{J}_{k}}\left|a_{n}\right|+\sum_{n \in \widetilde{J}_{k}}\left|a_{n}\right|\right)^{q} \\
& \leq C \sum_{k \in N_{\text {inter }}^{q}}\left(\# F_{k}\right)^{q-1} \sum_{l \in F_{k}}\left(\sum_{n \in I_{l}}\left|a_{n}\right|\right)^{q} \\
& +C \sum_{k \in N_{\text {inter }}^{q}}\left(\sum_{n \in \check{J}_{k}}\left|a_{n}\right|\right)^{q}+C \sum_{k \in N_{\text {inter }}^{q}}\left(\sum_{n \in \widehat{J}_{k}}\left|a_{n}\right|\right)^{q} \\
& \leq C M^{q-1} \sum_{l \in N_{\text {inter }}^{\mathcal{U}} \cup N_{\text {small }}^{\mathcal{S}}}\left(\sum_{n \in I_{l}}\left|a_{n}\right|\right)^{q} \\
& +C \sum_{k \in N_{\text {inter }}^{\mathcal{F}}}\left(\sum_{n \in I_{\phi(k)}}\left|a_{n}\right|\right)^{q}+\sum_{k \in N_{\text {inter }}^{\mathcal{q}}}\left(\sum_{n \in I_{\Phi(k)}}\left|a_{n}\right|\right)^{q} \\
& \leq C\left(\|a\|_{1, q}^{\mathcal{g}}\right)^{q} .
\end{aligned}
$$

Combining the above estimates, we conclude this implication.

Corollary 19. Let $1 \leq p<q \leq \infty$ and $\mathscr{I}, \mathscr{J}$ be such that

$$
\ell^{\mathscr{F}}(p, q) \subseteq \ell^{\mathscr{I}}(p, q) \Longleftrightarrow \sup \left\{\# \widetilde{G}_{l} ; l \in \mathbb{N}_{0}\right\}<\infty .
$$

The next result can be achieved using duality, but we include a direct proof.

Theorem 20. Let $1 \leq q<p \leq \infty$ and $\mathscr{F}, \mathscr{J}$ be such that

$$
\ell^{\mathscr{I}}(p, q) \subseteq \ell^{\mathcal{I}}(p, q) \Longleftrightarrow \sup \left\{\# \widetilde{F}_{k} ; k \in \mathbb{N}_{0}\right\}<\infty .
$$

Proof. $\Rightarrow$ ) Repeat the argument presented in the direct implication of Theorem 18.

$\Leftarrow)$ Denote again $\sup _{k}\left(\# F_{k}\right)=M$.

Case $p=\infty$. Observe first that if $l \in N_{\text {big }}^{\mathcal{J}} \cup N_{\text {equal }}^{\mathcal{I}}$, we have

$$
\left(\sup _{n \in I_{l}}\left|a_{n}\right|\right)^{q}=\left|a_{n(l)}\right|^{q} \leq\left(\sup _{n \in J_{k}}\left|a_{n}\right|\right)^{q}
$$

for some $k=k(l) \in N_{\text {small }}^{\mathscr{g}} \cup N_{\text {equal. }}^{\mathscr{g}}$. Since $k(l) \neq k\left(l^{\prime}\right)$ for $l \neq l^{\prime} \in$ $N_{\text {big }}^{\mathcal{S}} \cup N_{\text {equal }}^{\mathcal{J}}$, we obtain

$$
\sum_{l \in N_{\text {big }}^{\mathcal{J}} \cup N_{\text {equal }}^{\mathcal{J}}}\left(\sup _{n \in I_{l}}\left|a_{n}\right|\right)^{q} \leq \sum_{k \in N_{\text {small }}^{\mathcal{f}} \cup N_{\text {equal }}^{\mathcal{f}}}\left(\sup _{n \in J_{k}}\left|a_{n}\right|\right)^{q} .
$$

Also if $l \in N_{\text {inter }}^{\mathscr{J}}$, then $\left(\sup _{n \in I_{l}}\left|a_{n}\right|\right)^{q}=\left|a_{n(l)}\right|^{q}$ where $n(l) \epsilon$ $I_{l}^{\prime} \cup \widehat{I}_{l} \cup \check{I}_{l}$. Note that $n(l) \in J_{k}$ for some $k \in N_{\text {small }}^{\mathcal{F}} \cup N_{\text {inter }}^{\mathcal{F}}$ and

$$
1 \leq \#\left(\left\{l \in N_{\text {inter }}^{\mathcal{J}}: n(l) \in J_{k}\right\}\right) \leq 2 .
$$

Hence

$$
\sum_{l \in N_{\text {inter }}^{\mathcal{G}}}\left(\sup _{n \in I_{l}}\left|a_{n}\right|\right)^{q} \leq 2 \sum_{k \in N_{\text {small }}^{\mathcal{U}} \cup N_{\text {inter }}^{\mathcal{F}}}\left(\sup _{n \in J_{k}}\left|a_{n}\right|\right)^{q} .
$$

On the other hand,

$$
\begin{aligned}
\sum_{l \in N_{\text {small }}^{g}}\left(\sup _{n \in I_{l}}\left|a_{n}\right|\right)^{q} & \leq \sum_{k \in N_{\text {big }}^{\mathcal{f}} \cup N_{\text {inter }}^{\mathcal{g}}} \sum_{\psi(l)=k}\left(\sup _{n \in I_{l}}\left|a_{n}\right|\right)^{q} \\
& \leq \sum_{k \in N_{\text {big }}^{f} \cup N_{\text {inter }}^{\mathcal{g}}}\left(\sup _{n \in J_{k}}\left|a_{n}\right|\right)^{q}\left(\# F_{k}\right)^{q} \\
& \leq M^{q}\left(\|a\|_{p, \infty}^{\mathcal{g}}\right)^{q} .
\end{aligned}
$$

Combining the previous cases, we get $\ell^{\mathscr{f}}(\infty, q) \subseteq$ $\ell^{\mathcal{J}}(\infty, q)$.

Case $p<\infty$. Arguing as in Proposition 11, we simply show that $\ell^{\mathscr{f}}(p, 1) \subseteq \ell^{\mathcal{I}}(p, 1)$ for $p>1$. Consider

$$
\begin{aligned}
\|a\|_{p, 1}^{\mathcal{J}}= & \sum_{l}\left(\sum_{n \in I_{l}}\left|a_{n}\right|^{p}\right)^{1 / p} \\
\leq & \sum_{l \in N_{\text {small }}^{\mathcal{S}}}\left(\sum_{n \in I_{l}}\left|a_{n}\right|^{p}\right)^{1 / p} \\
& +\sum_{l \in N_{\text {equal }}^{\mathcal{S}} \cup N_{\text {big }}^{f}}\left(\sum_{k \in G_{l}} \sum_{n \in J_{k}}\left|a_{n}\right|^{p}\right)^{1 / p} \\
& +\sum_{l \in N_{\text {inter }}^{\mathcal{S}}}\left(\sum_{k \in G_{l}} \sum_{n \in J_{k}}\left|a_{n}\right|^{p}\right. \\
= & I_{1}+I_{2}+I_{3} .
\end{aligned}
$$


Now observe that

$$
\begin{aligned}
I_{1} & \leq \sum_{k \in N_{\text {big }}^{\mathcal{f} \cup N_{\text {inter }}^{\mathcal{g}}}} \sum_{l \in F_{k}}\left(\sum_{n \in I_{l}}\left|a_{n}\right|^{p}\right)^{1 / p} \\
& \leq \sum_{k \in N_{\text {big }}^{\mathcal{f}} \cup N_{\text {inter }}^{\mathcal{g}}}\left(\sum_{n \in J_{k}}\left|a_{n}\right|^{p}\right)^{1 / p} \#\left(F_{k}\right) \leq M\|a\|_{p, 1}^{\mathcal{g}} .
\end{aligned}
$$

Also note, since $p>1$,

$$
I_{2} \leq \sum_{l \in N_{\text {equal }}^{\mathcal{J}} \cup N_{\text {big }}^{\mathcal{J}}} \sum_{k \in G_{l}}\left(\sum_{n \in J_{k}}\left|a_{n}\right|^{p}\right)^{1 / p} \leq\|a\|_{p, 1}^{\mathcal{g}} .
$$

Finally

$$
\begin{aligned}
I_{3} \leq & \sum_{l \in N_{\text {inter }}^{\mathcal{g}}}\left(\sum_{k \in G_{l}} \sum_{n \in J_{k}}\left|a_{n}\right|^{p}\right)^{1 / p} \\
& +\left(\sum_{n \in \check{I}_{l}}\left|a_{n}\right|^{p}\right)^{1 / p}+\left(\sum_{n \in \widehat{I}_{l}}\left|a_{n}\right|^{p}\right)^{1 / p} \\
\leq & \sum_{k \in N_{\text {inter }}^{\mathcal{F}} \cup N_{\text {small }}^{\mathcal{F}}}\left(\sum_{n \in J_{k}}\left|a_{n}\right|^{p}\right)^{1 / p} \\
& +\sum_{l \in N_{\text {inter }}^{\mathcal{F}}}\left(\sum_{n \in J_{\psi(l)}}\left|a_{n}\right|^{p}\right)^{1 / p} \\
& +\sum_{l \in N_{\text {inter }}^{\mathcal{F}}}\left(\sum_{n \in J_{\Psi(l)}}\left|a_{n}\right|^{p}\right)^{1 / p} \\
\leq & C \sum_{k}\left(\sum_{n \in J_{k}}\left|a_{n}\right|^{p}\right)^{1 / p}=C\|a\|_{p, 1}^{\mathcal{g}} .
\end{aligned}
$$

The converse implication is now complete.

Corollary 21. Let $1 \leq q<p \leq \infty$ and $\mathscr{I}, \mathscr{J}$ be collections of intervals such that

$$
\ell^{\mathscr{I}}(p, q) \subseteq \ell^{\mathscr{I}}(p, q) \Longleftrightarrow \sup \left\{\# \widetilde{G}_{l} ; l \in \mathbb{N}_{0}\right\}<\infty .
$$

Corollary 22. Let $1 \leq p, q \leq \infty$ with $p \neq q$ and $\mathscr{I}, \mathscr{J}$ be collections of intervals such that

$$
\ell^{\mathscr{I}}(p, q)=\ell^{\mathscr{I}}(p, q) \Longleftrightarrow \sup \left\{\left(\# \widetilde{F}_{k}\right)\left(\# \widetilde{G}_{l}\right) ; k, l \in \mathbb{N}_{0}\right\}<\infty \text {. }
$$

Proof. It suffices to show the case $p<q$. Note that $\ell^{\mathscr{I}}(p, q) \subseteq$ $\ell^{\mathscr{F}}(p, q)$ and $\ell^{\mathscr{F}}(p, q) \subseteq \ell^{\mathscr{I}}(p, q)$ are equivalent, due to Theorem 18 and Corollary 19 , to the facts $\sup _{k}\left(\# \widetilde{F}_{k}\right)<\infty$ and $\sup _{l}\left(\# \widetilde{G}_{l}\right)<\infty$, or equivalently

$$
\sup \left\{\left(\# \widetilde{F}_{k}\right)\left(\# \widetilde{G}_{l}\right) ; k, l \in \mathbb{N}_{0}\right\}=\sup _{k}\left(\# \widetilde{F}_{k}\right) \sup _{l}\left(\# \widetilde{G}_{l}\right)<\infty \text {. }
$$

\section{Multipliers on Generalized Mixed Norm Spaces}

In this section, we consider $1 \leq r, s, u, v \leq \infty$ and $\mathscr{I}, \mathscr{J}$ such that $\Lambda_{\mathscr{I}}=\Lambda_{\mathscr{g}}$. We define

$$
\begin{aligned}
& \left(\ell^{\mathcal{I}}(r, s), \ell^{\mathscr{F}}(u, v)\right) \\
& =\left\{\lambda=\left(\lambda_{n}\right)_{n \in \Lambda_{\mathcal{F}} \cap \Lambda_{\mathcal{F}}}:\left\|\left(\lambda_{n} a_{n}\right)_{n \in \Lambda_{\mathcal{F}}}\right\|_{\mathcal{u}, v}^{\mathcal{F}}\right. \\
& \left.\leq C\left\|\left(a_{n}\right)_{n \in \Lambda_{\mathcal{F}}}\right\|_{r, s}^{\mathcal{F}}\right\} .
\end{aligned}
$$

The case $\mathscr{I}=\mathscr{J}$ can be shown repeating the proof for $\mathscr{I}=\left\{I_{k}: k \in \mathbb{N}_{0}\right\}$ where $I_{k}=\left[2^{k}-1,2^{k+1}-1\right) \cap \mathbb{N}_{0}$ (see $[1$, Theorem 1]).

Theorem 23. Consider $1 \leq r, s, u, v \leq \infty$. Then

$$
\left(\ell^{\mathcal{I}}(r, s), \ell^{\mathcal{I}}(u, v)\right)=\ell^{\mathcal{I}}(u \ominus r, v \ominus s) .
$$

We define the Köthe dual $\ell^{\mathcal{I}}(p, q)^{K}=\left(\ell^{\mathcal{I}}(p, q)\right.$, $\left.\ell^{\mathcal{F}}(1,1)\right)$.

Corollary 24. Consider $1 \leq r, s, u, v \leq \infty$. Then

$$
\ell^{\mathscr{I}}(r, s)^{K}=\ell^{\mathscr{I}}\left(r^{\prime}, s^{\prime}\right)
$$

There are some other cases where the set of multipliers can be easily determined. Using Proposition 6 and Corollary 22, one easily obtains the following results.

Proposition 25. (i) If $\sup _{k \in \mathbb{N}_{0}} \# J_{k}<\infty$, then $\left(\ell^{\mathcal{I}}(r, s)\right.$, $\left.\ell^{\mathscr{F}}(u, v)\right)=\ell^{\mathscr{I}}(v \ominus r, v \ominus s)$.

(ii) If $\sup _{l \in \mathbb{N}_{0}} \# I_{l}<\infty$, then $\left(\ell^{\mathscr{I}}(r, s), \ell^{\mathscr{F}}(u, v)\right)=\ell^{\mathscr{F}}(u \ominus$ $s, v \ominus s)$.

(iii) If $\sup \left\{\left(\# \widetilde{F}_{k}\right)\left(\# \widetilde{G}_{l}\right) ; k, l \in \mathbb{N}_{0}\right\}<\infty$, then

$$
\left(\ell^{\mathscr{I}}(r, s), \ell^{\mathscr{I}}(u, v)\right)=\ell^{\mathscr{I}}(u \ominus r, v \ominus s)=\ell^{\mathscr{I}}(u \ominus r, v \ominus s) .
$$

Also as a direct consequence of Theorem 18 we obtain the following.

Proposition 26. If $r \leq u, s \leq v$, and $u<v$ and $\sup \left\{\# \widetilde{F}_{k} ; k \in\right.$ $\left.\mathbb{N}_{0}\right\}<\infty$, then

$$
\left(\ell^{\mathscr{I}}(r, s), \ell^{\mathscr{F}}(u, v)\right)=\left\{\left(\lambda_{n}\right)_{n \in \Lambda_{\mathscr{I}}}: \sup _{n}\left|\lambda_{n}\right|<\infty\right\} .
$$

Proof. If $\lambda=\left(\lambda_{n}\right)_{n}$ is a multiplier then the sequence $\left(\lambda_{n}\right)$ is necessarily bounded.

$$
\left\{\left(\lambda_{n}\right)_{n \in \Lambda_{\mathcal{F}}}: \sup _{n}\left|\lambda_{n}\right|<\infty\right\} \subseteq\left(\ell^{\mathscr{I}}(r, s), \ell^{\mathscr{F}}(u, v)\right)
$$

is equivalent to $\ell^{\mathcal{I}}(r, s) \subseteq \ell^{\mathscr{J}}(u, v)$. Now use the embedding $\ell^{\mathscr{I}}(r, s) \subseteq \ell^{\mathscr{I}}(u, v)$ and Theorem 18 to conclude the result. 
Definition 27. Given two families of intervals, $\mathscr{I}, \mathscr{J}$, such that $\Lambda_{\mathscr{I}}=\Lambda_{\mathscr{J}}$, one defines the collection of pairwise disjoint intervals in $\mathbb{N}_{0}$ as

$$
\widetilde{\mathscr{I} \cap \mathscr{J}}=\left\{I_{l} \cap J_{k}: k \in \mathbb{N}_{0}, l \in \widetilde{F}_{k}\right\} .
$$

It coincides with $\left\{I_{l} \cap J_{k}: l \in \mathbb{N}_{0}, k \in \widetilde{G}_{l}\right\}$.

Proposition 28. Let $1 \leq r, s, u, v \leq \infty$.

(i) If $r \leq s, v \leq u$, then $\left(\ell^{\mathscr{I}}(r, s), \ell^{\mathscr{F}}(u, v)\right) \subseteq \ell^{\overline{\mathscr{I} \cap \mathscr{F}}}(u \ominus$ $r, v \ominus s)$.

In particular, if $\sup _{k} \# \widetilde{F}_{k}<\infty$, then

$$
\left(\ell^{\mathcal{I}}(r, s), \ell^{\mathscr{F}}(u, v)\right) \subseteq \ell^{\mathscr{I}}(u \ominus r, v \ominus s) .
$$

(ii) If $s \leq r, u \leq v$, then $e^{\overline{\mathscr{I \cap \mathscr { F }}}}(u \ominus r, v \ominus s) \subseteq$ $\left(\ell^{\mathcal{I}}(r, s), \ell^{\mathscr{f}}(u, v)\right)$.

In particular, if $\sup _{l} \# \widetilde{G}_{l}<\infty$, then

$$
\ell^{\mathscr{I}}(u \ominus r, v \ominus s) \subseteq\left(\ell^{\mathscr{I}}(r, s), \ell^{\mathscr{I}}(u, v)\right) .
$$

Proof. (i) Note that $\overline{\mathscr{I} \cap \mathscr{J}} \leq \mathscr{I}$ and $\overline{\mathscr{I} \cap \mathscr{J}} \leq \mathscr{J}$. Hence, from Proposition 11,

$$
\begin{aligned}
& \ell^{\overline{I^{\prime I}}}(p, q) \subseteq \ell^{\mathscr{I}}(p, q), \quad p \geq q, \\
& \ell^{\mathscr{F}}(p, q) \subseteq \ell^{\overline{\mathscr{I} \cap \mathscr{I}}}(p, q), \quad p \leq q .
\end{aligned}
$$

Now using (72) and Theorem 23, we obtain

$$
\begin{aligned}
& \left(\ell^{\mathscr{I}}(r, s), \ell^{\mathscr{I}}(u, v)\right) \subseteq\left(e^{\overline{\mathscr{F} \cap \mathscr{F}}}(r, s), \ell^{\overline{\mathscr{I} \cap \mathscr{I}}}(u, v)\right) \\
& =\ell^{\overline{\mathscr{F} \cap \mathscr{I}}}(u \ominus r, v \ominus s) .
\end{aligned}
$$

Also we have

$$
\begin{gathered}
F_{k}(\widetilde{\mathscr{I} \cap \mathscr{J}}, \mathscr{J})=\left\{l \in \mathbb{N}_{0}: I_{l} \cap J_{k} \subseteq J_{k}\right\}=\widetilde{F}_{k}, \\
G_{l}(\widetilde{\mathscr{I} \cap \mathscr{J}}, \mathscr{I})=\left\{k \in \mathbb{N}_{0}: I_{l} \cap J_{k} \subseteq I_{l}\right\}=\widetilde{G}_{l} .
\end{gathered}
$$

Using now Theorem 12,

$$
\begin{aligned}
& \ell^{\overline{\mathscr{I} \cap \mathcal{I}}}(p, q)=\ell^{\mathscr{I}}(p, q) \Longleftrightarrow \sup _{k} \# \widetilde{F}_{k}<\infty, \\
& \ell^{\widetilde{I \cap \mathscr{I}}}(p, q)=\ell^{\mathscr{I}}(p, q) \Longleftrightarrow \sup _{l} \# \widetilde{G}_{l}<\infty .
\end{aligned}
$$

The particular case follows now applying (75).

(ii) is similar to the previous one and left to the reader.

Our purpose is to get a final description of multipliers $\left(\ell^{\mathscr{I}}(r, s), \ell^{\mathscr{F}}(u, v)\right)$. We will deal first with the case $\mathscr{I} \leq \mathscr{J}$ and get a reduction to this situation in the remaining cases.
3.1. The Case $\mathscr{I} \leq \mathscr{J}$. In this section, we consider $\mathscr{I}$ and $\mathscr{J}$ such that $\mathbb{N}_{0}=N_{\text {big }}^{\mathscr{f}} \cup N_{\text {equal }}^{\mathscr{f}}$. This means that $\widetilde{F}_{k}=F_{k} \neq \emptyset$ and $J_{k}=\cup_{l \in F_{k}} I_{l}$ for all $k$. Notice that $l \in F_{k}$ means $I_{l} \subseteq J_{k}$ and we have

$$
F_{k}=\left\{l \in \mathbb{N}_{0}: \phi(k) \leq l \leq \Phi(k)\right\} .
$$

We use the notation $\mathscr{J} / \mathscr{I}=\left\{F_{k}: k \in \mathbb{N}_{0}\right\}$.

We will need the following well-known fact.

Lemma 29. Let $0<u, r \leq \infty, A \subseteq \mathbb{N}_{0}$, and $\left(\lambda_{i}\right)_{i \in A}$. There exists $\left(a_{i}\right)_{i \in A}$ such that

$$
\begin{gathered}
\left(\sum_{i \in A}\left|a_{i}\right|^{r}\right)^{1 / r}=1 \\
\left(\sum_{i \in A}\left|\lambda_{i}\right|^{u \ominus r}\right)^{1 / u \ominus r}=\left(\sum_{i \in A}\left|a_{i} \lambda_{i}\right|^{u}\right)^{1 / u}
\end{gathered}
$$

(with the obvious modifications whenever $u, r$ or $u \ominus r$ equals $\infty)$.

Proof. For $r=\infty$ (then $u \ominus r=u$ ), it suffices to take $a_{i}=1, i \in$ A.

If $r<\infty$ and $u \geq r$ (hence $u \ominus r=\infty$ ), it suffices to take

$$
a_{i}= \begin{cases}1 & i=i(A) \\ 0 & \text { otherwise }\end{cases}
$$

for $i(A)$ such that $\sup _{i \in A}\left|\lambda_{i}\right|=\left|\lambda_{i(A)}\right|$.

If $u<r<\infty$, take

$$
a_{i}=\left(\sum_{i \in A}\left|\lambda_{i}\right|^{u \ominus r}\right)^{-1 / r} \lambda_{i}^{u \ominus r / r}, \quad i \in A .
$$

Using $1+u \ominus r / r=u \ominus r / u$, one shows the result.

Theorem 30. If $\mathscr{I} \leq \mathscr{J}$, then

$$
\begin{aligned}
& \left(\ell^{\mathcal{I}}(r, s), \ell^{\mathcal{F}}(u, v)\right) \\
& \quad=\left\{\left(\lambda_{n}\right)_{n}:\left(\left(\sum_{i \in I_{l}}\left|\lambda_{i}\right|^{u \Theta r}\right)^{1 / u \ominus r}\right)_{l}\right.
\end{aligned}
$$

$$
\left.\in \ell^{\mathscr{f} / \mathscr{I}}(u \ominus s, v \ominus s)\right\} .
$$

Proof. $\subseteq)$ Assume that $\left(\lambda_{n}\right)_{n} \in\left(\ell^{\mathcal{I}}(r, s), \ell^{\mathscr{F}}(u, v)\right)$.

We use Lemma 29 with $A=I_{l}$ to select for each $l \in \mathbb{N}_{0}$ a sequence $\left(a_{i}^{(l)}\right)_{i \in I_{l}}$ such that $\left(\sum_{i \in I_{l}}\left|a_{i}^{(l)}\right|^{r}\right)^{1 / r}=1$ and $\beta_{l}=$ $\left(\sum_{i \in I_{l}}\left|\lambda_{i}\right|^{u \ominus r}\right)^{1 / u \ominus r}=\left(\sum_{i \in I_{l}}\left|\lambda_{i} a_{i}^{(l)}\right|^{u}\right)^{1 / u}$.

Now, again use Lemma 29 with $A=F_{k}$ for each $k \epsilon$ $\mathbb{N}_{0}$ to choose $\left(\alpha_{l}\right)_{l \in F_{k}}$ verifying $\left(\sum_{l \in F_{k}}\left|\alpha_{l}\right|^{s}\right)^{1 / s}=1$ and $\left(\sum_{l \in F_{k}} \beta_{l}^{u \ominus s}\right)^{1 / u \ominus s}=\left(\sum_{l \in F_{k}}\left|\beta_{l} \alpha_{l}\right|^{u}\right)^{1 / u}$. 
Finally, using Lemma 29 for $A=\mathbb{N}_{0}$, one more time, take $\gamma=\left(\gamma_{k}\right)_{k}$ verifying $\left(\sum_{k}\left|\gamma_{k}\right|^{s}\right)^{1 / s}=1$ and

$\left(\sum_{k}\left(\sum_{l \in F_{k}} \beta_{l}^{u \ominus s}\right)^{v \ominus s / u \ominus s}\right)^{1 / v \ominus s}=\left(\sum_{k} \gamma_{k}^{v}\left(\sum_{l \in F_{k}} \beta_{l}^{u \ominus s}\right)^{v / u \ominus s}\right)^{1 / v}$.

This procedure allows us to obtain the sequence $a=\left(a_{i}\right)_{i}$, $a_{i}=\gamma_{k} \alpha_{l} a_{i}^{(l)}$ where $i \in I_{l}, l \in F_{k}$, and $k \in \mathbb{N}_{0}$. With this choice, we get that $\|a\|_{r, s}^{\mathscr{F}}=1$ and

$$
\|\beta\|_{u \ominus s, v \ominus s}^{\mathscr{F} / \mathscr{F}}=\|\lambda * a\|_{u, v}^{\mathscr{F}} \leq\|\lambda\| .
$$

つ) Let $a=\left(a_{i}\right)_{i} \in \ell^{\mathcal{J}}(r, s)$ and $\lambda=\left(\lambda_{i}\right)_{i}$ such that $\left(\beta_{l}\right)_{l} \in$ $\ell^{\mathscr{F} / \mathcal{F}}(u \ominus s, v \ominus s)$ where

$$
\beta_{l}=\left(\sum_{i \in I_{l}}\left|\lambda_{i}\right|^{u \ominus r}\right)^{1 / u \ominus r} .
$$

Fix $k \in \mathbb{N}_{0}$ :

$$
\begin{aligned}
\left(\sum_{i \in J_{k}}\left|\lambda_{i} a_{i}\right|^{u}\right)^{1 / u} & =\left(\sum_{l \in F_{k}} \sum_{i \in I_{l}}\left|\lambda_{i} a_{i}\right|^{u}\right)^{1 / u} \\
& \leq\left(\sum_{l \in F_{k}}\left(\sum_{i \in I_{l}}\left|\lambda_{i}\right|^{u \ominus r}\right)^{u / u \ominus r}\left(\sum_{i \in I_{l}}\left|a_{i}\right|^{r}\right)^{u / r}\right)^{1 / u} \\
& \leq\left(\sum_{l \in F_{k}}\left(\sum_{i \in I_{l}}\left|\lambda_{i}\right|^{u \ominus r}\right)^{u \ominus s / u \ominus r}\right)^{1 / u \ominus s} \\
& \times\left(\sum_{l \in F_{k}}\left(\sum_{i \in I_{l}}\left|a_{i}\right|^{r}\right)^{s / r}\right)^{1 / s} .
\end{aligned}
$$

Taking the $v$-norm, we get

$$
\begin{aligned}
\left(\sum_{k}\left(\sum_{i \in J_{k}}\left|\lambda_{i} a_{i}\right|^{u}\right)^{v / u}\right)^{1 / v} & \left(\sum_{k}\left(\sum_{l \in F_{k}} \beta_{l}^{u \ominus s}\right)^{v / u \ominus s}\left(\sum_{l \in F_{k}}\left(\sum_{i \in I_{l}}\left|a_{i}\right|^{r}\right)^{s / r}\right)^{v / s}\right)^{1 / v} \\
\leq & \left(\sum_{k}\left(\sum_{l \in F_{k}} \beta_{l}^{u \ominus s}\right)^{v \ominus s / u \ominus s}\right)^{1 / v \ominus s} \\
& \times\left(\sum_{k} \sum_{l \in F_{k}}\left(\sum_{i \in I_{l}}\left|a_{i}\right|^{r}\right)^{s / r}\right)^{1 / s} \\
= & \left(\sum_{k}\left(\sum_{l \in F_{k}} \beta_{l}^{u \ominus s}\right)^{v \ominus s / u \ominus s}\right)^{1 / v \ominus s} \\
& \times\left(\sum_{l}\left(\sum_{i \in I_{l}}\left|a_{i}\right|^{r}\right)^{s / r}\right)^{1 / s} .
\end{aligned}
$$

Hence $\left(\lambda_{n}\right)_{n} \in\left(\ell^{\mathcal{I}}(r, s), \ell^{\mathscr{F}}(u, v)\right)$ and $\|\lambda\| \leq\|\beta\|_{u \ominus s, v \ominus s}^{\mathscr{F} / \mathscr{I}}$.
Corollary 31. Let $\mathscr{J} \leq \mathscr{I}$ and $1 \leq r, s, u, v \leq \infty$. Then

$$
\begin{gathered}
\left(\ell^{\mathcal{F}}(r, s), \ell^{\mathcal{F}}(u, v)\right) \\
=\left\{\left(\lambda_{n}\right)_{n}:\left(\left(\sum_{i \in J_{k}}\left|\lambda_{i}\right|^{u \ominus r}\right)^{1 / u \ominus r}\right)_{k}\right. \\
\left.\in \ell^{\mathcal{F} / \mathcal{F}}(v \ominus r, v \ominus s)\right\} .
\end{gathered}
$$

Proof. Recall that $\widetilde{G}_{l}=G_{l}=\left\{k \in \mathbb{N}_{0}: J_{k} \subseteq I_{l}\right\}$ and $I_{l}=$ $\cup_{k \in G_{l}} J_{k}$. We now denote $\mathscr{I} / \mathscr{J}=\left\{G_{l}: l \in \mathbb{N}_{0}\right\}$. Using Köthe duals. we actually have

$$
\left(\ell^{\mathcal{F}}(r, s), \ell^{\mathcal{F}}(u, v)\right)=\left(\ell^{\mathcal{F}}\left(u^{\prime}, v^{\prime}\right), \ell^{\mathcal{F}}\left(r^{\prime}, s^{\prime}\right)\right) .
$$

Taking into account that $p^{\prime} \ominus q^{\prime}=q \ominus p$ for all $p, q$, the result follows from Theorem 30 .

3.2. The Case $\overline{\mathscr{I} \cap \mathscr{J}} \subseteq \mathscr{I} \cup \mathscr{F}$. Let $\mathscr{I}=\left\{I_{l}: l \in \mathbb{N}_{0}\right\}$ and $\mathscr{J}=\left\{J_{k}: k \in \mathbb{N}_{0}\right\}$ be such that $\Lambda_{\mathscr{F}}=\Lambda_{\mathscr{f}}$. We assume in this section that $N_{\text {inter }}^{\mathcal{J}}=\emptyset$ and $N_{\text {inter }}^{\mathscr{\mathcal { T }}}=\emptyset$, that is to say, for a given $l \in \mathbb{N}_{0}$ either there exists $k$ such that $I_{l} \subseteq J_{k}$ or there exists $k^{\prime}$ such that $J_{k^{\prime}} \subseteq I_{l}$. In other words, each interval in $\overline{\mathscr{I} \cap \mathscr{J}}$ belongs either to $\mathscr{I}$ or to $\mathscr{F}$.

To extend the result on multipliers to this setting we will use the following lemma whose easy proof is left to the reader.

Lemma 32. Let $\mathscr{I}=\left\{I_{l}: l \in \mathbb{N}_{0}\right\}$ and $\mathscr{J}=\left\{J_{k}: k \in \mathbb{N}_{0}\right\}$ be such that $\Lambda_{\mathscr{I}}=\Lambda_{\mathscr{f}}$ and let $\mathscr{I}_{i}$ (respect. $\mathscr{F}_{i}$ ) be subcollections of $\mathscr{I}$ (respect. $\mathscr{J}$ ) for $i=1, \ldots, m$ where $\mathscr{I}=\cup_{i=1}^{m} \mathscr{I}_{i}$ (respect. $\left.\mathscr{J}=\cup_{i=1}^{m} \mathscr{F}_{i}\right)$ satisfying $\Lambda_{\mathscr{I}_{i}}=\Lambda_{\mathscr{F}_{i}}$ for $i=1, \ldots, m$. Then

$$
\lambda=\left(\lambda_{n}\right)_{n \in \Lambda_{\mathcal{I}}} \in\left(\ell^{\mathcal{F}}(r, s), \ell^{\mathcal{F}}(u, v)\right)
$$

if and only if

$$
\lambda^{(i)}=\left(\lambda_{n}\right)_{n \in \Lambda_{\mathscr{S}_{i}}} \in\left(\ell^{\mathscr{S}_{i}}(r, s), \ell^{\mathscr{f}_{i}}(u, v)\right), \quad i=1, \ldots, m .
$$

$$
\text { Moreover }\|\lambda\| \approx \sum_{i=1}^{m}\left\|\lambda^{(i)}\right\| .
$$

Theorem 33. Let $\overline{\mathscr{I} \cap \mathscr{J}} \subseteq \mathscr{I} \cup \mathcal{F}$. Then $\left(\lambda_{n}\right)_{n} \in$ $\left(\ell^{\mathcal{J}}(r, s), \ell^{\mathcal{F}}(u, v)\right)$ if and only if it satisfies the conditions

$$
\begin{gathered}
\left(\left(\sum_{i \in J_{k}}\left|\lambda_{i}\right|^{u \ominus r}\right)^{1 / u \ominus r}\right)_{k \in N_{\text {equal }}^{\mathcal{F}}} \in \ell^{v \ominus s}, \\
\left(\left(\sum_{i \in I_{l}}\left|\lambda_{i}\right|^{u \ominus r}\right)^{1 / u \ominus r}\right)_{l \in N_{\text {small }}^{\mathcal{S}}} \in \ell^{\mathscr{F}}(u \ominus s, v \ominus s), \\
\left(\left(\sum_{i \in J_{k}}\left|\lambda_{i}\right|^{u \ominus r}\right)^{1 / u \ominus r}\right)_{k \in N_{\text {small }}^{\mathcal{F}}} \in \ell^{\mathcal{G}}(v \ominus r, v \ominus s),
\end{gathered}
$$

where $\mathscr{F}=\left\{F_{k}: k \in N_{b i g}^{\mathscr{F}}\right\}$ and $\mathscr{G}=\left\{G_{l}: l \in N_{b i g}^{\mathscr{F}}\right\}$. 
Proof. Let us consider the following collection of intervals:

$$
\begin{aligned}
& \mathscr{J}_{b}=\left\{J_{k}: k \in N_{\text {big }}^{\mathcal{J}}\right\}, \\
& \mathscr{J}_{e}=\left\{J_{k}: k \in N_{\text {equal }}^{\mathcal{F}}\right\}, \\
& \mathscr{J}_{s}=\left\{J_{k}: k \in N_{\text {small }}^{\mathcal{F}}\right\}
\end{aligned}
$$

and similarly for $\mathscr{I}$.

If $J_{k} \in \mathscr{J}_{b}$ (respect. $I_{l} \in \mathscr{I}_{b}$ ), we have $F_{k}=\left\{l \in \mathbb{N}_{0}: I_{l} \varsubsetneqq\right.$ $\left.J_{k}\right\} \neq \emptyset$ (respect. $G_{l}=\left\{k \in \mathbb{N}_{0}: J_{k} \varsubsetneqq I_{l}\right\} \neq \emptyset$ ) and

$$
J_{k}=\cup_{l \in F_{k}} I_{l}, \quad I_{l} \in \mathscr{I}_{s} \quad \text { (respect. } I_{l}=\cup_{l \in G_{l}} J_{k}, J_{k} \in \mathscr{J}_{s} \text { ). }
$$

Hence $\mathscr{F}=\mathscr{J}_{e} \cup \mathscr{J}_{b} \cup \mathscr{J}_{s}, \mathscr{I}=\mathscr{I}_{e} \cup \mathscr{I}_{b} \cup \mathscr{I}_{s}$, and

$$
\mathscr{J}_{e}=\left\{J_{k}: k \in N_{\text {equal }}^{\mathcal{F}}\right\}=\left\{I_{l}: l \in N_{\text {equal }}^{\mathcal{I}}\right\}=\mathscr{I}_{e} \text {. }
$$

Observe that $\mathscr{I}_{s} \leq \mathscr{J}_{b}$ and $\mathscr{J}_{s} \leq \mathscr{I}_{b}$ and, in particular, $\mathscr{G}=$ $\mathscr{I}_{b} / \mathscr{J}_{s}$ and $\mathscr{F}=\mathscr{J}_{b} / \mathscr{I}_{s}$.

We use Lemma 32 and observe that, denoting $\Lambda_{0}=\Lambda_{\mathscr{F}_{e}}$, $\Lambda_{1}=\Lambda_{\mathscr{F}_{b}}=\Lambda_{\mathscr{I}_{s}}$, and $\Lambda_{2}=\Lambda_{\mathscr{F}_{s}}=\Lambda_{\mathscr{F}_{b}}$,

$$
\left(\lambda_{n}\right)_{n \in \Lambda_{0}} \in\left(\ell^{\mathscr{F}_{e}}(r, s), \ell^{\mathscr{F}_{e}}(u, v)\right)
$$

corresponds to (90) invoking Theorem 23, also that

$$
\left(\lambda_{n}\right)_{n \in \Lambda_{1}} \in\left(\ell^{\mathscr{F}_{s}}(r, s), \ell^{\mathscr{F}_{b}}(u, v)\right)
$$

corresponds to (91) invoking Theorem 30, and, finally, that

$$
\left(\lambda_{n}\right)_{n \in \Lambda_{2}} \in\left(\ell^{\mathscr{I}_{b}}(r, s), \ell^{\mathscr{F}_{s}}(u, v)\right)
$$

corresponds to (92) invoking Corollary 31.

3.3. The General Case. In this section, we assume that there exist $k \in \mathbb{N}_{0}$ and $l \in \widetilde{F}_{k}$ such that $I_{l} \cap J_{k} \in \widetilde{\mathscr{I} \cap \mathscr{J}}$ and $I_{l} \cap J_{k} \notin$ $\mathscr{I} \cup \mathcal{J}$.

Since the notation may be a bit confusing, we will illustrate the idea. Let $\mathscr{I}, \mathscr{J}$ be different partitions of $\mathbb{N}_{0}$ as shown in Figure 2.

The situation we are handling now corresponds to $N_{\text {inter }}^{\mathscr{J}} \neq \emptyset\left(\right.$ and hence $\left.N_{\text {inter }}^{\mathcal{I}} \neq \emptyset\right)$.

Definition 34. Consider

$$
\begin{aligned}
& \mathscr{J}^{\prime}=\left\{J_{k}^{\prime}=\cup_{l \in F_{k}} I_{l}: k \in \mathbb{N}_{0}, \# F_{k}>0\right\}, \\
& \mathscr{H}=\widetilde{\mathscr{I} \cap \mathscr{J}} \backslash(\mathscr{I} \cup \mathscr{J}), \\
& \mathscr{J}_{s}=\left\{J_{k}: k \in N_{\text {small }}^{\mathscr{F}}\right\} .
\end{aligned}
$$

Denote $\mathscr{J}^{\prime \prime}=\mathscr{J}^{\prime} \cup \mathscr{J}_{s}$ and $\mathscr{J}_{\text {new }}=\mathscr{J}^{\prime \prime} \cup \mathscr{H}$.

We use similar notations for $\mathscr{I}$.

Recalling that $\phi(k)=\min \widetilde{F}_{k}$ and $\Phi(k)=\max \widetilde{F}_{k}$ for $k \in \mathbb{N}_{0}$, we easily observe that $\phi\left(N_{\text {equal }}^{\mathscr{J}}\right) \subseteq N_{\text {equal }}^{\mathscr{I}}, \phi\left(N_{\text {big }}^{\mathscr{J}}\right) \subseteq$ $N_{\text {small }}^{\mathcal{I}}, \phi\left(N_{\text {small }}^{\mathscr{I}}\right) \subseteq N_{\text {big }}^{\mathscr{I}} \cup N_{\text {inter }}^{\mathscr{I}}$, and $\phi\left(N_{\text {inter }}^{\mathcal{I}}\right) \subseteq N_{\text {small }}^{\mathcal{I}} \cup N_{\text {inter }}^{\mathscr{I}}$. Same results hold for $\Phi$.
Lemma 35. Consider the families defined in the previous definition. Then:

$$
\begin{aligned}
\mathscr{H} & =\left\{\widehat{J}_{k}: k \in N_{\text {inter }}^{\mathscr{g}}, \phi(k) \in N_{\text {inter }}^{\mathcal{I}}\right\} \\
& \cup\left\{\check{J}_{k}: k \in N_{\text {inter }}^{\mathcal{g}}, \Phi(k) \in N_{\text {inter }}^{\mathcal{I}}\right\} .
\end{aligned}
$$

Proof. $\subseteq$ ) Let $I \in \mathscr{H}$. Since $I \in \overline{\mathscr{I} \cap \mathscr{J}}$, then there exist $k \in \mathbb{N}_{0}$ and $l \in \widetilde{F}_{k}$ such that $I=I_{l} \cap J_{k}$. On the other hand, since $I \notin \mathscr{I} \cup \mathscr{J}$, we have that $I \subsetneq I_{l}$ and $I \subsetneq J_{k}$. Hence, either $\phi(k)=l$ and $\psi(l)=k$ or $\Phi(k)=l$ and $\psi(l)=k$. This gives either $k \in N_{\text {inter }}^{\mathscr{J}}$ and $\phi(k) \in N_{\text {inter }}^{\mathscr{J}}$ (and hence $I=\widehat{J}_{k}$ ) or $k \in N_{\text {inter }}^{\mathscr{J}}$ and $\Phi(k) \in N_{\text {inter }}^{\mathscr{I}}$ (and hence $I=\check{J}_{k}$ ).

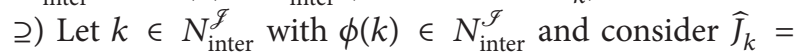

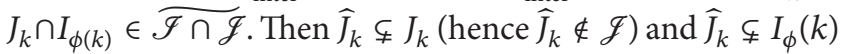
(hence $\left.\widehat{J}_{k} \notin \mathscr{I}\right)$. One may proceed similarly for $\breve{J}_{k}$ in the case $k \in N_{\text {inter }}^{\mathscr{J}}$ with $\Phi(k) \in N_{\text {inter }}^{\mathscr{I}}$.

Remark 36. Note that $\widehat{J}_{k}=J_{k} \cap I_{l}$ if and only if $\check{I}_{l}=I_{l} \cap J_{k}$. Therefore,

$$
\begin{gathered}
\mathscr{H}=\left\{\widehat{I}_{l}: l \in N_{\text {inter }}^{\mathscr{I}}, \psi(l) \in N_{\text {inter }}^{\mathscr{I}}\right\} \\
\cup\left\{\check{I}_{l}: k \in N_{\text {inter }}^{\mathcal{I}}, \Psi(l) \in N_{\text {inter }}^{\mathscr{J}}\right\} .
\end{gathered}
$$

Lemma 37. Consider the different families defined in Definition 34. Then:

$$
\widetilde{\mathscr{I}^{\prime \prime} \cap \mathscr{J}^{\prime \prime}} \subseteq \mathscr{I}_{s} \cup \mathscr{J}_{s} \cup \mathscr{I}_{e} \subseteq \mathscr{I}^{\prime \prime} \cup \mathscr{J}^{\prime \prime} .
$$

Proof. Let $I \in \mathscr{I}^{\prime} \cup \mathscr{I}_{s}$ and $J \in \mathscr{J}^{\prime} \cup \mathscr{J}_{s}$ with $I \cap J \neq \emptyset$. The case $I \in \mathscr{I}_{s}$ and $J \in \mathscr{J}_{s}$ can not hold. If $I \in \mathscr{I}_{s}$ and $J \in \mathscr{J}^{\prime}$, then $I \cap J=I \in \mathscr{I}_{s}$. Similarly if $I \in \mathscr{I}^{\prime}$ and $J \in \mathscr{J}_{s}$, then $I \cap J=J \in \mathscr{J}_{s}$. Finally if $I \in \mathscr{I}^{\prime}$ and $J \in \mathscr{J}^{\prime}$, then $I=J \in$ $\mathscr{I}_{e}=\mathscr{J}_{e}$

Theorem 38. $\lambda \in\left(\ell^{\mathcal{I}}(r, s), \ell^{\mathscr{I}}(u, v)\right)$ if and only if $\left(\lambda_{n}\right)_{n}$ satisfies

$$
\begin{aligned}
& \left(\left(\sum_{i \in J_{k}}\left|\lambda_{i}\right|^{u \ominus r}\right)^{1 / u \ominus r}\right)_{k \in N_{\text {equal }}^{\mathcal{g}}} \in \ell^{v \ominus s} \\
& \left(\left(\sum_{i \in I_{l}}\left|\lambda_{i}\right|^{u \ominus r}\right)^{1 / u \ominus r}\right)_{l \in N_{\text {small }}^{\mathscr{F}}} \in \ell^{\mathscr{F}}(u \ominus s, v \ominus s) \\
& \left(\left(\sum_{i \in J_{k}}\left|\lambda_{i}\right|^{u \ominus r}\right)^{1 / u \ominus r}\right)_{k \in N_{\text {small }}^{\mathcal{F}}} \in \ell^{\mathscr{G}}(v \ominus r, v \ominus s) \\
& \left(\left(\sum_{i \in \check{J}_{k}}\left|\lambda_{i}\right|^{u \ominus r}\right)^{1 / u \ominus r}\right)_{k \in \Lambda_{r}} \\
& +\left(\left(\sum_{i \in \widehat{J}_{k}}\left|\lambda_{i}\right|^{u \ominus r}\right)^{1 / u \ominus r}\right)_{k \in \Lambda_{l}} \in \ell^{v \ominus s,}
\end{aligned}
$$




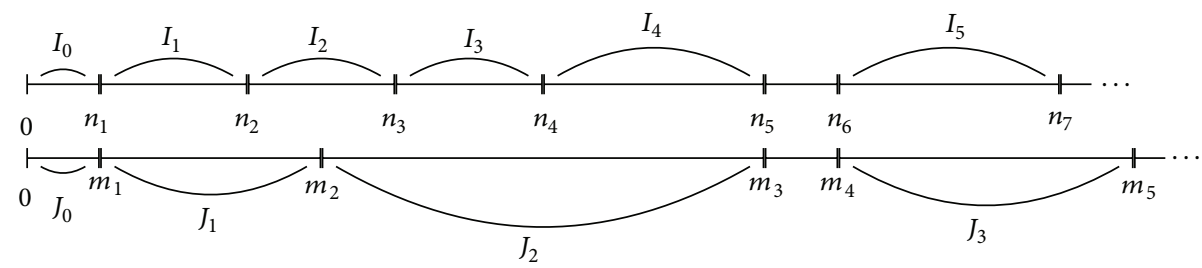

Figure 2

where

$$
\begin{aligned}
& \Lambda_{r}=\left\{k \in N_{\text {inter }}^{\mathcal{g}}, \Phi(k) \in N_{\text {inter }}^{\mathcal{I}}\right\}, \\
& \Lambda_{l}=\left\{k \in N_{\text {inter }}^{\mathscr{I}}, \phi(k) \in N_{\text {inter }}^{\mathcal{I}}\right\}, \\
& \mathscr{G}=\left\{G_{l}: l \in N_{\text {big }}^{\mathcal{I}} \cup N_{\text {inter }}^{\mathcal{I}}, \# G_{l}>0\right\}, \\
& \mathscr{F}=\left\{F_{k}: k \in N_{\text {big }}^{\mathcal{I}} \cup N_{\text {inter }}^{\mathcal{F}}, \# F_{k}>0\right\} .
\end{aligned}
$$

Proof. Using $J_{k}=J_{k}^{\prime} \cup \widehat{J}_{k} \cup \check{J}_{k}$ and Lemma 35, one obtains $\mathscr{J}_{\text {new }} \leq \mathscr{J}$ and $\mathscr{I}_{\text {new }} \leq \mathscr{I}$. Clearly, $\# F_{l}\left(\mathscr{I}_{\text {new }}, \mathscr{I}\right) \leq 3$ and $\# F_{k}\left(\mathscr{J}_{\text {new }}, \mathscr{J}\right) \leq 3$ for all $k$. Therefore, using Theorem 12, we have $\ell^{\mathscr{F} \text { new }}(p, q)=\ell^{\mathscr{I}}(p, q)$ and $\ell^{\mathscr{I}_{\text {new }}}(p, q)=\ell^{\mathscr{I}}(p, q)$, which gives

$$
\left(\ell^{\mathscr{I}}(r, s), \ell^{\mathscr{F}}(u, v)\right)=\left(\ell^{\mathcal{F}_{\text {new }}}(r, s), \ell^{\mathscr{f} \text { new }}(u, v)\right)
$$

Taking into account Lemma 35 and Remark 36, we observe that $\Lambda_{\mathscr{H}}=\Lambda_{r} \cup \Lambda_{l}$ and $\Lambda_{\mathscr{I}^{\prime \prime}}=\Lambda_{\mathscr{g}^{\prime \prime}}$.

Since $\mathscr{J}_{\text {new }}=\mathscr{J}^{\prime \prime} \cup \mathscr{H}$ and $\mathscr{I}_{\text {new }}=\mathscr{I}^{\prime \prime} \cup \mathscr{H}$, we can apply Lemma 32 to conclude that $\lambda \in\left(\ell^{\mathscr{I}}(r, s), \ell^{\mathscr{J}}(u, v)\right)$ if and only if $\left(\lambda_{n}\right)_{n \in \Lambda_{\mathscr{H}}} \in\left(\ell^{\mathscr{H}}(r, s), \ell^{\mathscr{H}}(u, v)\right)$ and $\left(\lambda_{n}\right)_{n \notin \Lambda_{\mathscr{H}}} \in$ $\left(\ell^{\mathcal{F}^{\prime \prime}}(r, s), \ell^{\mathcal{F}^{\prime \prime}}(u, v)\right)$.

Now apply Theorem 23 to obtain $\left(\lambda_{n}\right)_{n \in \Lambda_{\mathscr{H}}} \in \ell^{\mathscr{H}}(u \ominus r, v \ominus$ s) which corresponds to (106).

On the other hand, comparing $\mathscr{I}^{\prime \prime}$ and $\mathscr{J}^{\prime \prime}$, we notice that $I \in \mathscr{I}_{\text {big }}^{\prime \prime}$ corresponds to $I=I_{l}^{\prime}$ for some $l \in N_{\text {big }}^{\mathscr{J}} \cup N_{\text {inter }}^{\mathscr{J}}$ and $\# G_{l} \geq 1$. Hence we obtain that $\mathscr{G}=\left\{G_{I}: I \in \mathscr{I}_{\text {big }}^{\prime \prime}\right\}$ and similarly $\mathscr{F}=\left\{F_{J}: J \in \mathscr{J}_{\text {big }}^{\prime \prime}\right\}$.

We now use Lemma 37 together with Theorem 33 to obtain the equivalence with (103), (104), and (105) and $\left(\lambda_{n}\right)_{n \notin \Lambda_{\mathscr{C}}} \in\left(\ell^{\mathcal{F}^{\prime \prime}}(r, s), \ell^{\mathcal{F}^{\prime \prime}}(u, v)\right)$.

\section{An Application}

Let $\rho:[0,1) \rightarrow[0, \infty)$ be a nondecreasing function such that $\rho(0)=0$ and $\rho(t) / t \in L^{1}([0,1))$; we define the weighted Bergman-Besov space $B^{1}(\rho)$ as those analytic functions $F$ in the unit disk such that

$$
\int_{\mathbb{D}}\left|F^{\prime}(z)\right| \frac{\rho(1-|z|)}{1-|z|} d A(z)<\infty .
$$

An analytic function $F$ is called lacunary if $F(z)=$ $\sum_{n \in \Lambda_{\mathscr{L}}} a_{n} z^{n}$ where $\mathscr{L}=\left\{\left\{n_{k}\right\}: k \in \mathbb{N}_{0}\right\}$ for some $\left(n_{k}\right)$ such that $\inf _{k} n_{k+1} / n_{k}>1$.
Recently, weights with the following condition had been considered in [16]: there exist $C_{1}, C_{2}>0$ and $K(n, \rho)$ such that

$$
\begin{aligned}
& C_{1} \int_{0}^{1} r^{2^{n}-1} \frac{\rho(1-r)}{1-r} d r \leq K(n, \rho) \\
& \quad \leq C_{2} \int_{1-2^{-n}}^{1-2^{-(n+1)}} r^{2^{n+1}-1} \frac{\rho(1-r)}{1-r} d r,
\end{aligned}
$$

and the following result has been shown.

Theorem 39 (see [16]). Let $F(z)=\sum_{n \in \Lambda_{\mathscr{L}}} a_{n} z^{n}$ be a lacunary function and let $\rho$ be a weight satisfying (110). Then $F$ belongs to $B^{1}(\rho)$ if and only if

$$
\sum_{k=0}^{\infty}\left(\sum_{n \in J_{k}}\left|a_{n}\right|^{2}\right)^{1 / 2} 2^{k} K(k, \rho)<\infty,
$$

where $J_{k}=\left\{n: 2^{k}-1 \leq n<2^{k+1}-1\right\}$.

We will extend the previous result for more general classes of weight functions and families of intervals $\mathscr{J}$.

Definition 40. Let $0<q<\infty$; let $\mathscr{J}$ be a collection of disjoint intervals in $\mathbb{N}_{0}$, say $J_{k}=\mathbb{N}_{0} \cap\left[m_{k}, m_{k+1}\right)$, where $m_{0}=0$ and $\left(m_{k}\right)$ is some increasing sequence in $\mathbb{N}_{0}$. And let $\rho:[0,1) \rightarrow$ $[0, \infty)$ be a measurable function such that $\rho(t) / t \in L^{1}([0,1))$.

We say that $\rho$ is $q$-adapted to $\mathscr{J}$ whenever there exists $C$ > 0 depending on $m_{n}, q$ and $\rho$ such that

$$
\int_{0}^{1} r^{q m_{n}} \frac{\rho(1-r)}{1-r} d r \leq C \int_{A_{n}} r^{q m_{n+1}} \frac{\rho(1-r)}{1-r} d r
$$

for all $n \geq 0$ where $A_{0}=\left[0,1-1 / m_{1}\right)$ and $A_{n}=\left[1-1 / m_{n}, 1-\right.$ $\left.1 / m_{n+1}\right)$ for $n \geq 1$.

We denote

$$
\mu_{\rho}(s)=\int_{0}^{1} r^{s} \frac{\rho(1-r)}{1-r} d r, \quad s \geq 0
$$

In particular, from condition (112) if $\rho$ is $q$-adapted to $\mathscr{J}$, we get that

$$
\mu_{\rho}\left(q m_{n}\right) \approx \mu_{\rho}\left(q m_{n+1}\right) .
$$

Note also that condition (110) means that $\rho$ is $1 / 2$-adapted for $\mathscr{J}$ where $m_{n}=2^{n}-1$. 
Proposition 41. Let $\rho_{\alpha}(t)=t^{\alpha}$ with $\alpha>0$ and $\mathscr{J}=$ $\left\{\left[m_{n}, m_{n+1}\right) \cap \mathbb{N}_{0}: n \in \mathbb{N}_{0}\right\}$. The following statements are equivalent:

(i) $\rho_{\alpha}$ is $q$-adapted to $\mathscr{J}$ for all $q>0$,

(ii) $\rho_{\alpha}$ is q-adapted to $\mathscr{J}$ for some $q>0$,

(iii) $\sup _{n} m_{n+1} / m_{n}<\infty$.

Proof. (i) $\Rightarrow$ (ii) It is trivial.

(ii) $\Rightarrow$ (iii) It is well known that $B(n+1, \alpha)=\int_{0}^{1} r^{n}(1-$ $r)^{\alpha-1} d r \approx n^{-\alpha}$ and therefore $\mu_{\rho_{\alpha}}\left(q m_{n}\right) \approx m_{n}^{-\alpha}$.

Hence it follows from (114) that $m_{n+1} \approx m_{n}$. Therefore $\sup m_{n+1} / m_{n}<\infty$.

(iii) $\Rightarrow$ (i) Let $\sup m_{n+1} / m_{n}=\delta$ and take $q>0$. Now observe that

$$
\begin{aligned}
& \int_{1-1 / m_{n}}^{1-1 / m_{n+1}} r^{q m_{n+1}}(1-r)^{\alpha-1} d r \\
& \quad \geq\left(1-\frac{1}{m_{n}}\right)^{q m_{n+1}} \int_{1 / m_{n+1}}^{1 / m_{n}} s^{\alpha-1} d s \\
& \quad \geq \frac{1}{\alpha}\left(1-\frac{1}{m_{n}}\right)^{q m_{n+1}} m_{n}^{-\alpha}\left(1-\left(\frac{m_{n}}{m_{n+1}}\right)^{\alpha}\right) \\
& \geq \frac{1}{\alpha}\left(\left(1-\frac{1}{m_{n}}\right)^{m_{n}}\right)^{\delta q} m_{n}^{-\alpha}\left(1-\frac{1}{\delta^{\alpha}}\right) \\
& \geq C \mu_{\rho_{\alpha}}\left(q m_{n}\right) .
\end{aligned}
$$

We now modify the proof of Lemma 3 in [14] to obtain the following result.

Lemma 42. Let $0<q \leq 1$, let $\mathcal{J}$ be a collection of disjoint intervals in $\mathbb{N}_{0}$, and assume $\rho$ is a weight q-adapted to $\mathscr{J}$. If $\left(\alpha_{n}\right) \geq 0$, then

$$
\int_{0}^{1}\left(\sum_{n=0}^{\infty} \alpha_{n} r^{n}\right)^{q} \frac{\rho(1-r)}{1-r} d r \approx \sum_{n=0}^{\infty}\left(\sum_{k \in J_{n}} \alpha_{k}\right)^{q} \mu_{\rho}\left(q m_{n}\right)
$$

where $J_{n}=\left\{k: m_{n} \leq k<m_{n+1}\right\}$.

Proof. As above, $A_{0}=\left[0,1-1 / m_{1}\right)$ and $A_{n}=\left[1-1 / m_{n}, 1-\right.$ $\left.1 / m_{n+1}\right)$ for $n \geq 1$. Then

$$
\begin{aligned}
& \int_{0}^{1}\left(\sum_{n=0}^{\infty} \alpha_{n} r^{n}\right)^{q} \frac{\rho(1-r)}{1-r} d r \\
& \quad=\sum_{n=0}^{\infty} \int_{A_{n}}\left(\sum_{n=0}^{\infty} \alpha_{n} r^{n}\right)^{q} \frac{\rho(1-r)}{1-r} d r \\
& \geq \sum_{n=0}^{\infty} \int_{A_{n}}\left(\sum_{k \in J_{n}} \alpha_{k} r^{k}\right)^{q} \frac{\rho(1-r)}{1-r} d r
\end{aligned}
$$

$$
\begin{aligned}
& \geq \sum_{n=0}^{\infty} \int_{A_{n}}\left(\sum_{k \in J_{n}} \alpha_{k}\right)^{q} r^{q m_{n+1}} \frac{\rho(1-r)}{1-r} d r \\
& \geq C^{-1} \sum_{n=0}^{\infty}\left(\sum_{k \in J_{n}} \alpha_{k}\right)^{q} \mu_{\rho}\left(q m_{n}\right) .
\end{aligned}
$$

Conversely, since $q \leq 1$,

$$
\begin{aligned}
& \int_{0}^{1}\left(\sum_{n=0}^{\infty} \alpha_{n} r^{n}\right)^{q} \frac{\rho(1-r)}{1-r} d r \\
& \quad \leq \int_{0}^{1} \sum_{n=0}^{\infty}\left(\sum_{k \in J_{n}} \alpha_{k} r^{k}\right)^{q} \frac{\rho(1-r)}{1-r} d r \\
& \leq \sum_{n=0}^{\infty}\left(\sum_{k \in J_{n}} \alpha_{k}\right)^{q}\left(\int_{0}^{1} r^{q m_{n}} \frac{\rho(1-r)}{1-r} d r\right) \\
& \quad \leq \sum_{n=0}^{\infty}\left(\sum_{k \in J_{n}} \alpha_{k}\right)^{q} \mu_{\rho}\left(q m_{n}\right) .
\end{aligned}
$$

We first note that for lacunary functions $F$ and $0<p<$ $\infty$, we have (see [17])

$$
\begin{aligned}
M_{p}(F, r) & =\left(\int_{0}^{2 \pi}\left|F\left(r e^{i \theta}\right)\right|^{p} \frac{d \theta}{2 \pi}\right)^{1 / p} \approx M_{2}(F, r) \\
& =\left(\int_{0}^{2 \pi}\left|F\left(r e^{i \theta}\right)\right|^{2} \frac{d \theta}{2 \pi}\right)^{1 / 2} .
\end{aligned}
$$

Therefore, for lacunary functions $F$, one has that $F \in B^{1}(\rho)$ if and only if

$$
\int_{0}^{1} M_{2}\left(F^{\prime}, r\right) \frac{\rho(1-r)}{1-r} d r<\infty
$$

Therefore, invoking Plancherel's theorem and Lemma 42, we recover Theorem 39.

Recall that an analytic function $F: \mathbb{D} \rightarrow \mathbb{C}$ with $F(z)=$ $\sum_{n=0}^{\infty} a_{n} z^{n}$ is said to belong to $H(p, q, \rho)$ (see [14, Definition 2]) whenever

$$
\|F\|_{H(p, q, \rho)}=\left(\int_{0}^{1} M_{p}^{q}(F, r) \frac{\rho\left(1-r^{2}\right)}{1-r^{2}} r d r\right)^{1 / q}<\infty .
$$

We use the notation $H(p, q, \alpha)$ if $\rho(t)=t^{\alpha}$.

A consequence of Lemma 42 is the following result.

Corollary 43. Let $0<q \leq 2$, let $\mathscr{J}$ be a collection of disjoint intervals in $\mathbb{N}_{0}$, and let $\rho$ be a weight $q / 2$-adapted to $\mathcal{F}$. Then

$$
\|F\|_{H(2, q, \rho)} \approx\left(\sum_{n=0}^{\infty}\left(\sum_{k \in J_{n}}\left|a_{k}\right|^{2}\right)^{q / 2} \mu_{\rho}\left(\frac{q m_{n}}{2}\right)\right)^{1 / q} .
$$


Moreover, if $F$ is lacunary and $0<p<\infty$, then

$$
\|F\|_{H(p, q, \rho)} \approx\left(\sum_{n=0}^{\infty}\left(\sum_{k \in J_{n} \cap \Lambda_{\mathscr{L}}}\left|a_{k}\right|^{2}\right)^{q / 2} \mu_{\rho}\left(\frac{q m_{n}}{2}\right)\right)^{1 / q} .
$$

Theorem 44. Let $0<q<\infty$, let $\mathscr{J}$ be a collection of disjoint intervals in $\mathbb{N}_{0}$, and assume $\rho$ is a weight q-adapted to $\mathscr{J}$. Define $\lambda=\left(\lambda_{k}\right)_{k}$ such that

$$
\lambda_{k}=\left(\int_{0}^{1} r^{q m_{n}} \frac{\rho(1-r)}{1-r}\right)^{1 / q}, \quad k \in J_{n}
$$

and $\lambda_{k}=0$ otherwise. Then $\left(\lambda_{k}\right)_{k} \in\left(H(1, q, \rho), \ell^{\mathscr{F}}(\infty, q)\right)$.

Proof. We will show that

$$
\left(\sum_{n=0}^{\infty}\left(\sup _{k \in J_{n}}\left|a_{k}\right|\right)^{q} \mu_{\rho}\left(q m_{n}\right)\right)^{1 / q} \leq C\|F\|_{H(1, q, \rho)} .
$$

Recall that

$$
\sup _{k \in J_{n-1}}\left|a_{k}\right| r^{k} \leq M_{1}(F, r)
$$

and therefore, if $A_{0}=\left[0,1-1 / m_{1}\right)$ and $A_{n}=\left[1-1 / m_{n}, 1-\right.$ $1 / m_{n+1}$ ) for $n \geq 1$, then

$$
\begin{aligned}
& \sum_{n=0}^{\infty}\left(\sup _{k \in J_{n}}\left|a_{k}\right|\right)^{q} \mu_{\rho}\left(q m_{n}\right) \\
& \quad \leq C \sum_{n=0}^{\infty}\left(\sup _{k \in J_{n}}\left|a_{k}\right|\right)^{q} \int_{A_{n}} r^{q m_{n+1}} \frac{\rho(1-r)}{1-r} d r \\
& \quad \leq C \sum_{n=0}^{\infty} \int_{A_{n}}\left(\sup _{k \in J_{n}}\left|a_{k}\right| r^{k}\right)^{q} \frac{\rho(1-r)}{1-r} d r \\
& \quad \leq C \sum_{n=0}^{\infty} \int_{A_{n}} M_{1}^{q}(F, r) \frac{\rho(1-r)}{1-r} d r \\
& \quad=C\|F\|_{H(1, q, \rho)}^{q} .
\end{aligned}
$$

Theorem 45. Let $1 \leq q_{2}<q_{1} \leq 2$ and let $\mathscr{J}$ and $\mathscr{I}$ be collections of disjoint intervals in $\mathbb{N}_{0}$, generated by sequences $m_{k}$ and $n_{k}$, respectively, such that $\mathscr{I} \leq \mathscr{J}$. Assume that $\rho_{1}$ is a weight $q_{1} / 2$-adapted to $\mathscr{I}$ and $\rho_{2}$ is a weight $q_{2} / 2$-adapted to J. Denote

$$
\begin{aligned}
& \mu_{\rho_{1}, \rho_{2}}(k) \\
& =\left(\left(\mu_{\rho_{2}}\left(\frac{q_{2} m_{k}}{2}\right)\right)^{1 / q_{2}}\left(\mu_{\rho_{1}}\left(\frac{q_{1} n_{k}}{2}\right)\right)^{-1 / q_{1}}\right)^{1 / q_{2} \ominus q_{1}}
\end{aligned}
$$

then

$$
\begin{aligned}
(H & \left.\left(2, q_{1}, \rho_{1}\right), H\left(2, q_{2}, \rho_{2}\right)\right) \\
\quad & =\left\{\left(\lambda_{n}\right)_{n} ;\left(\sup _{k \in I_{n}} \mu_{\rho_{1}, \rho_{2}}(k)\left|\lambda_{k}\right|\right) \in \ell^{\mathcal{F} / \mathscr{I}}\left(\infty, q_{2} \ominus q_{1}\right)\right\} .
\end{aligned}
$$

Proof. Let

$$
\begin{aligned}
& F_{\mathscr{I}}(z)=\sum_{k=0}^{\infty}\left(\mu_{\rho_{1}}\left(\frac{q_{1} n_{k}}{2}\right)\right)^{1 / q_{1}}\left(\sum_{j \in I_{k}} z^{j}\right), \\
& \widetilde{F}_{\mathscr{I}}(z)=\sum_{k=0}^{\infty}\left(\mu_{\rho_{1}}\left(\frac{q_{1} n_{k}}{2}\right)\right)^{-1 / q_{1}}\left(\sum_{j \in I_{k}} z^{j}\right), \\
& G_{\mathscr{J}}(z)=\sum_{k=0}^{\infty}\left(\mu_{\rho_{2}}\left(\frac{q_{2} m_{k}}{2}\right)\right)^{1 / q_{2}}\left(\sum_{j \in J_{k}} z^{j}\right) .
\end{aligned}
$$

Using Corollary 43, one has that $f \in H\left(2, q_{1}, \rho_{1}\right)$ if and only if $f * F_{\mathscr{J}} \in \ell^{\mathcal{I}}\left(2, q_{1}\right)$ and $g \in H\left(2, q_{2}, \rho_{2}\right)$ if and only if $g * G_{\mathscr{g}} \in$ $\ell^{\mathscr{F}}\left(2, q_{2}\right)$.

We use that $\lambda \in\left(H\left(2, q_{1}, \rho_{1}\right), H\left(2, q_{2}, \rho_{2}\right)\right)$ is equivalent to $\lambda * G_{\mathscr{f}} \in\left(H\left(2, q_{1}, \rho_{1}\right), \ell^{\mathscr{F}}\left(2, q_{2}\right)\right)$ and also equivalent to $\lambda * G_{\mathscr{J}} * \widetilde{F}_{\mathscr{I}} \in\left(\ell^{\mathscr{I}}\left(2, q_{1}\right), \ell^{\mathscr{I}}\left(2, q_{2}\right)\right)$.

Making use of Theorem 30, we have

$$
\begin{aligned}
& \left(\ell^{\mathscr{I}}\left(2, q_{1}\right), \ell^{\mathscr{I}}\left(2, q_{2}\right)\right) \\
& \quad=\left\{\left(\gamma_{n}\right)_{n} ;\left(\sup _{k \in I_{n}}\left|\gamma_{k}\right|\right)_{n} \in \ell^{\mathscr{F} / \mathscr{I}}\left(\infty, q_{2} \ominus q_{1}\right)\right\} .
\end{aligned}
$$

This concludes the result.

Let us finish by observing some examples to apply the above results.

Example 46. Let $\lambda>1$ and denote $m_{0}(\lambda)=0$ and $m_{k}(\lambda)=$ $\left[\lambda^{k}\right]$ for $k \in \mathbb{N}_{0}$ and $\mathscr{J}(\lambda)$ the partition of intervals $J_{k}(\lambda)=$ $\left[m_{k}(\lambda), m_{k+1}(\lambda)\right) \cap \mathbb{N}_{0}$. In this case, $\mu_{\rho_{\alpha}}\left(q m_{n}\right) \approx \lambda^{-\alpha n}$, and then, from Proposition 41, $\rho_{\alpha}$ is $q$-adapted to $\mathscr{J}(\lambda)$ for any value of $q>0$.

Let $\lambda>\gamma>1$ with $\lambda=\gamma^{N}$ with $N \in \mathbb{N}_{0}$. Then $\mathscr{J}(\gamma) \leq$ $\mathscr{J}(\lambda)$ because

$$
m_{k}(\lambda)=\left[\lambda^{k}\right]=\left[\gamma^{N k}\right]=m_{N k}(\gamma)
$$

and therefore

$$
J_{k}(\lambda)=\cup_{l \in F_{k}} J_{l}(\gamma)
$$

where $F_{k}=\{l: N k \leq l<N k+N\}$. Hence $\mathscr{J}(\lambda) / \mathscr{J}(\gamma)=\mathscr{I}$ where $I_{k}=[N k, N(k+1)) \cap \mathbb{N}_{0}$; that is, $m_{k}(\mathscr{I})=N k$.

\section{Conflict of Interests}

The authors declare that there is no conflict of interests regarding the publication of this paper.

\section{Acknowledgments}

The authors thank the referee for her comments on the paper. Oscar Blasco and Zaragoza-Berzosa have been partially supported by Spanish Project BMF2011-231674. 


\section{References}

[1] C. N. Kellogg, "An extension of the Hausdorff-Young theorem," The Michigan Mathematical Journal, vol. 18, no. 2, pp. 121-127, 1971.

[2] G. H. Hardy and J. E. Littlewood, "Some properties of fractional integrals. II," Mathematische Zeitschrift, vol. 34, no. 1, pp. 403439, 1932.

[3] G. H. Hardy and J. E. Littlewood, "Theorems concerning mean values of analytic or harmonic functions," The Quarterly Journal of Mathematics, vol. 12, pp. 221-256, 1941.

[4] T. M. Flett, "The dual of an inequality of Hardy and Littlewood and some related inequalities," Journal of Mathematical Analysis and Applications, vol. 38, pp. 746-765, 1972.

[5] T. M. Flett, "Lipschitz spaces of functions on the circle and the disc," Journal of Mathematical Analysis and Applications, vol. 39, pp. 125-158, 1972.

[6] T. M. Flett, "On the rate of growth of mean values of holomorphic and harmonic functions," Proceedings of the London Mathematical Society, vol. 20, pp. 749-768, 1970.

[7] W. T. Sledd, "Some results about spaces of analytic functions introduced by Hardy and Littlewood," Journal of the London Mathematical Society, vol. 2, pp. 328-336, 1974.

[8] W. T. Sledd, “On multipliers of $H^{p}$ spaces," Indiana University Mathematics Journal, vol. 27, no. 5, pp. 797-803, 1978.

[9] M. Pavlović, "Mixed norm spaces of analytic and harmonic functions. I," Publications de l'Institut Mathématique, vol. 40, no. 54, pp. 117-141, 1986.

[10] M. Pavlović, "Mixed norm spaces of analytic and harmonic functions. II," Publications de l'Institut Mathématique, vol. 41, no. 55, pp. 97-110, 1987.

[11] D. M. Campbell and R. J. Leach, "A survey of $H^{p}$ multipliers as related to classical function theory," Complex Variables, vol. 3, no. 1-3, pp. 85-111, 1984.

[12] B. Osikiewicz, "Multipliers of Hardy spaces," Quaestiones Mathematicae, vol. 27, no. 1, pp. 57-73, 2004.

[13] O. Blasco, "Operators on weighted Bergman spaces $(0<p \leq 1)$ and applications," Duke Mathematical Journal, vol. 66, no. 3, pp. 443-467, 1992.

[14] O. Blasco, "Multipliers on weighted Besov spaces of analytic functions," in Banach spaces, vol. 144 of Contemporary Mathematics, pp. 23-33, 1993.

[15] O. Blasco, "Multipliers on spaces of analytic functions," Canadian Journal of Mathematics, vol. 20, pp. 1-21, 1994.

[16] E. Kwessi, G. De Souza, A. Abebe, and R. Aulaskari, "Characterization of lacunary functions in weighted Bergman-BesovLipschitz spaces," Complex Variables and Elliptic Equations, vol. 58, no. 2, pp. 157-162, 2013.

[17] A. Zygmund, Trigonometric Series, vol. 1-2 of Cambridge Mathematical Library, Cambridge University Press, Cambridge, UK, 3rd edition, 2002. 


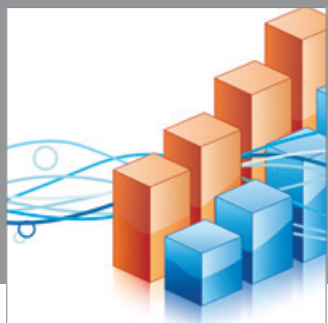

Advances in

Operations Research

mansans

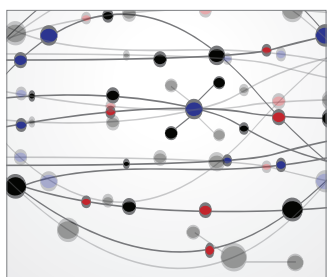

The Scientific World Journal
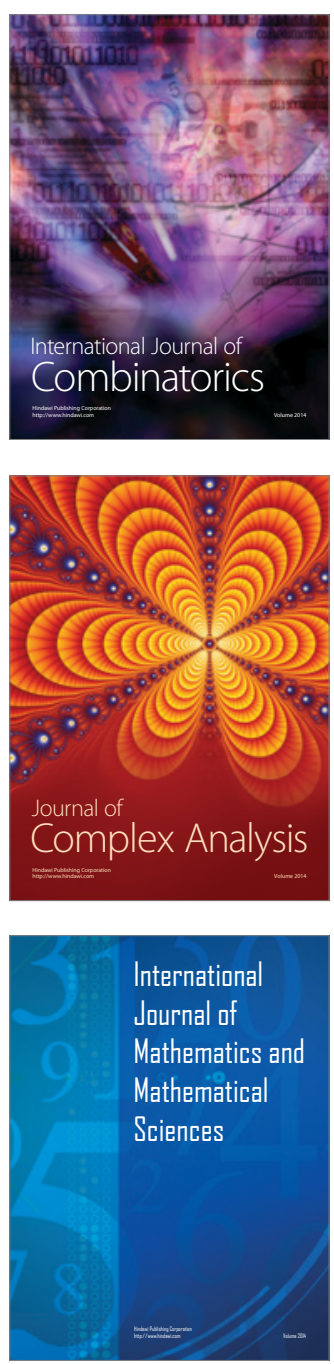
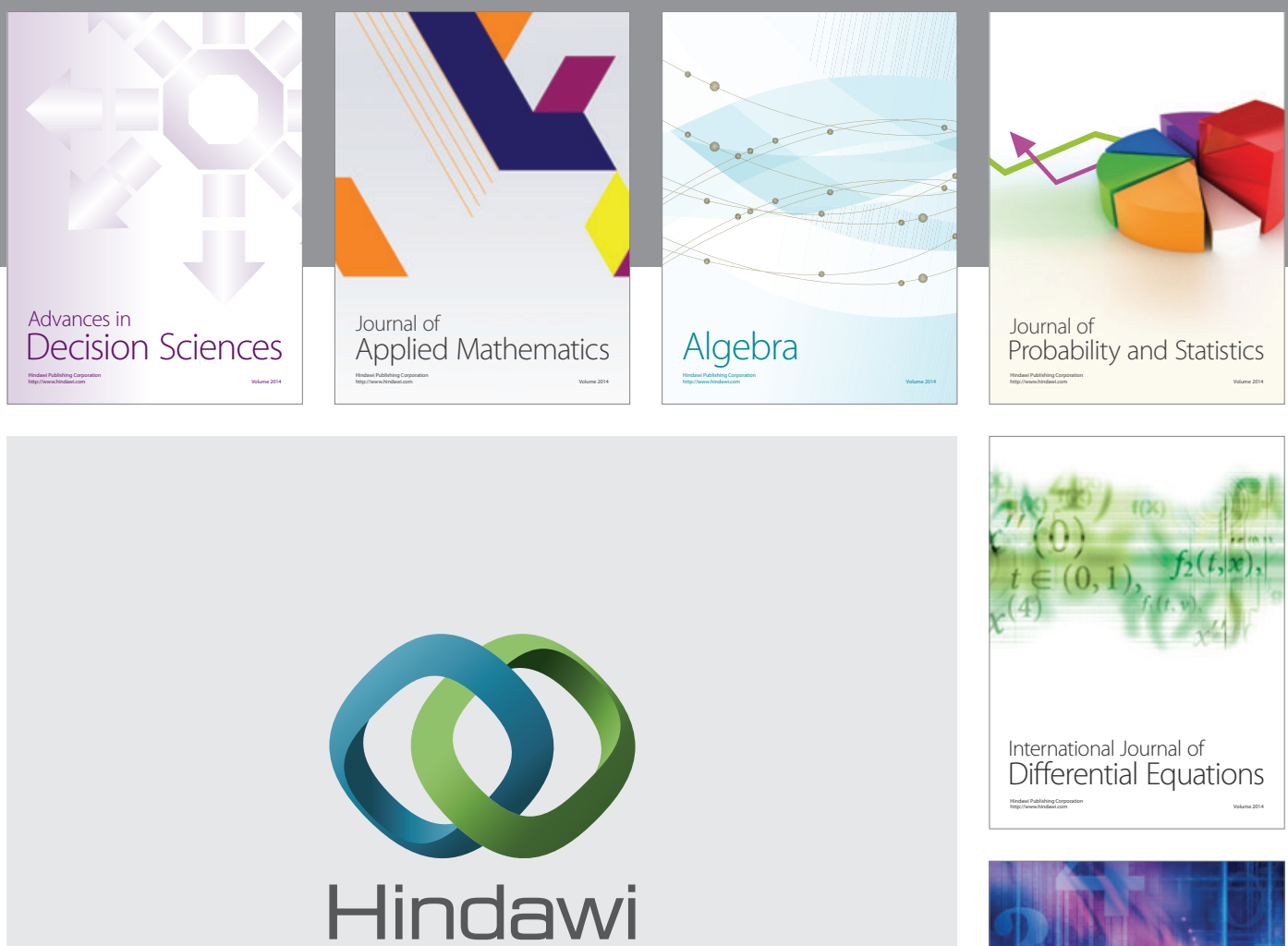

Submit your manuscripts at http://www.hindawi.com
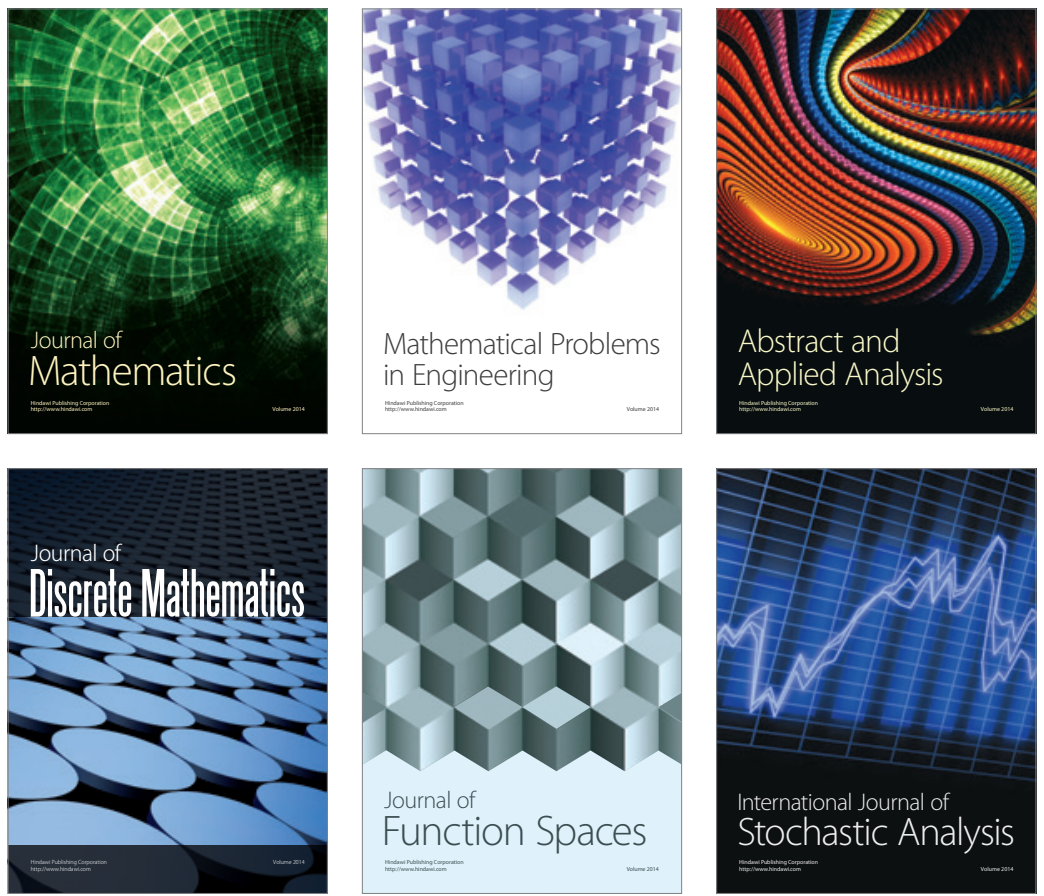

Journal of

Function Spaces

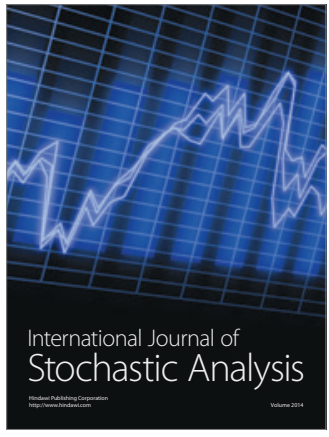

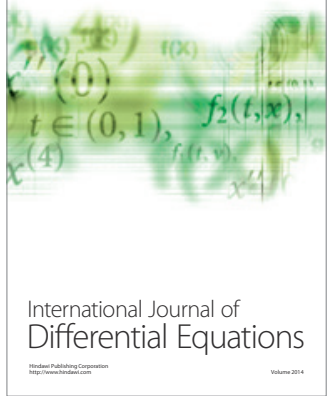
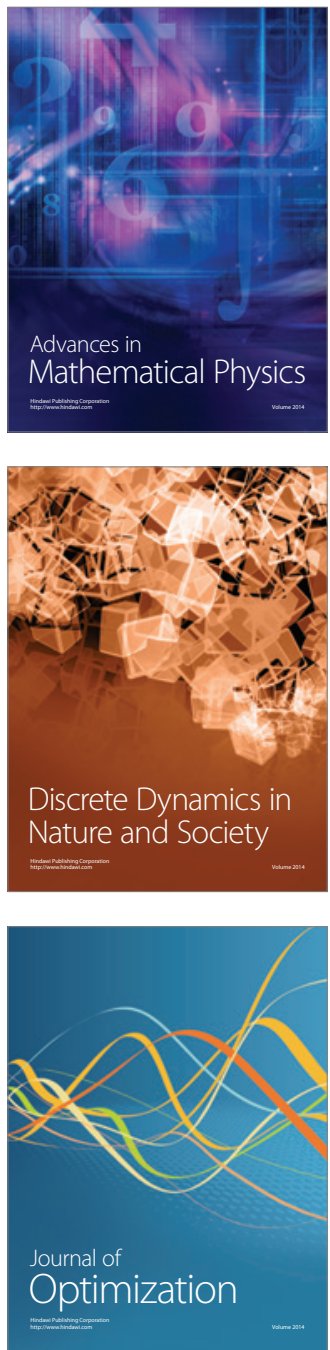